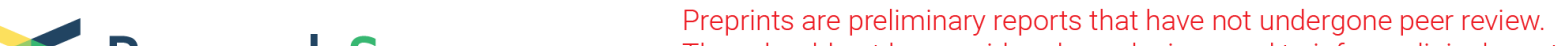 Research Square
or referenced by the media as validated information.
}

\section{Housing Conditions, Level of Feeding and Presence of Antibiotics in The Feed Shape Rabbit Cecal Microbiota}

María Velasco-Galilea ( $\nabla$ maria.velasco@irta.es )

Institut de Recerca i Tecnologia Agroalimentaries https://orcid.org/0000-0001-9533-7412

Miriam Guivernau

Institut de Recerca i Tecnologia Agroalimentaries

Miriam Piles

Institut de Recerca i Tecnologia Agroalimentaries

Marc Viñas

Institut de Recerca i Tecnologia Agroalimentaries

Oriol Rafel

Institut de Recerca i Tecnologia Agroalimentaries

Armand Sánchez

Universitat Autonoma de Barcelona

Yuliaxis Ramayo-Caldas

Institut de Recerca i Tecnologia Agroalimentaries

Olga González-Rodríguez

Institut de Recerca i Tecnologia Agroalimentaries

Juan P. Sánchez

Institut de Recerca i Tecnologia Agroalimentaries

\section{Research}

Keywords: cecal microbiota, meat rabbit, housing conditions, feed restriction, antibiotics, $16 \mathrm{~S}$ MiSeq Illumina sequencing, analysis of variance, multivariate approach

Posted Date: June 24th, 2020

DOl: https://doi.org/10.21203/rs.3.rs-36587/v1

License: (1) (1) This work is licensed under a Creative Commons Attribution 4.0 International License.

Read Full License 


\section{Housing conditions, level of feeding and presence of antibiotics in the feed shape rabbit cecal microbiota}

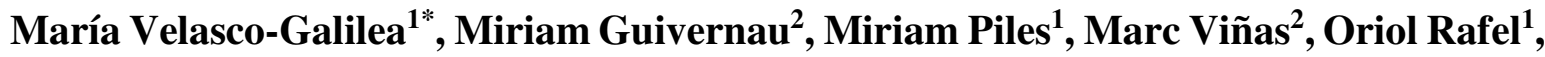
Armand Sánchez ${ }^{3,4}$, Yuliaxis Ramayo-Caldas ${ }^{1}$, Olga González-Rodríguez ${ }^{1}$ and Juan P. Sánchez ${ }^{1}$

${ }^{1}$ Institute for Food and Agriculture Research and Technology (IRTA) - Animal Breeding and Genetics, E08140 Caldes de Montbui, Barcelona, Spain,

${ }^{2}$ Institute for Food and Agriculture Research and Technology (IRTA) - Integral Management of Organic Waste, E08140 Caldes de Montbui, Barcelona, Spain,

${ }^{3}$ Animal Genomics Group, Centre for Research in Agricultural Genomics (CRAG) CSICIRTA-UAB-UB, Campus UAB, Catalonia, Spain,

${ }^{4}$ Unit of Animal Science, Department of Animal and Food Science, Autonomous University of Barcelona, Barcelona, Spain

*Corresponding author:

María Velasco-Galilea

maria.velasco@irta.es 


\section{Abstract}

2 Background: the effect of the production environment and different management practices

3 in rabbit cecal microbiota remains poorly understood. While previous studies have proved

4 the impact of the age or the feed composition, research in the housing conditions and other

5 animal management aspects, such as the presence of antibiotics in the feed or the level of

6 feeding, is still needed. Characterization of microbial diversity and composition of growing

7 rabbits raised under different conditions could help better understand the role these practices

8 play in cecal microbial communities and how it may result in different animal performance.

9 Results: four hundred twenty-five meat rabbits raised in two different facilities, fed under

10 two feeding regimes (ad libitum or restricted) with feed supplemented or free of antibiotics,

11 were selected for this study. A 16S rDNA-based assessment through the MiSeq Illumina

12 sequencing platform was performed on cecal samples collected from these individuals at

13 slaughter. Different univariate and multivariate approaches were conducted to unravel the

14 influence of the different factors on microbial alpha diversity and composition at phylum,

15 genus and OTU taxonomic levels. The animals raised in the facility harboring the most stable

16 environmental conditions had greater, and less variable, microbial richness and diversity.

17 Bootstrap univariate analyses of variance and sparse partial least squares-discriminant

18 analyses endorsed that the farm exerted the largest influence on rabbit microbiota since the

19 relative abundances of many taxa were found differentially represented between both

20 facilities at all taxonomic levels characterized. Furthermore, only five OTUs were needed to

21 achieve a perfect classification of samples according to the facility where animals were

22 raised. The level of feeding and the presence of antibiotics did not modify the global alpha

23 diversity but had an impact on some bacteria relative abundances, albeit in a small number 
24 of taxa compared with the farm, which is consistent with the lower sample classification

25 power according to these factors achieved using microbial information.

26 Conclusions: this study reveals different degrees of influence attributable to environment

27 and animal management. It highlights the importance of offering a controlled breeding

28 environment that reduces differences in microbial cecal composition that could be causative

29 of different animal performance.

\section{Keywords}

32 cecal microbiota, meat rabbit, housing conditions, feed restriction, antibiotics, 16S MiSeq

33 Illumina sequencing, analysis of variance, multivariate approach

\section{Background}

36 Microbial communities that inhabit the gastrointestinal tract (GIT) of animals constitute a

37 complex ecosystem whose members constantly interact between them and with their host [1-

38 Gaskins, 1997]. These interactions ensure homeostatic balance maintenance since GIT

39 ecosystem components are involved in many physiological and immunological processes [2-

40 Belkaid and Hand, 2014]. In the case of the domestic meat rabbit (Oryctolagus cuniculus), a

41 small herbivorous mammalian belonging to the family Leporidae, cecum is the main organ

42 for microbial fermentation. Thus, it is no surprising that rabbit cecum hosts the richest and

43 the most diverse microbial community of its GIT [3-Gouet and Fonty, 1979]. For this reason, 
44 cecum has been the organ preferably chosen in previous rabbit gut microbiota assessments

45 [4-Abecia et al., 2007; 5-Zou et al., 2016; 6-Zhu et al., 2017; 7-Chen et al., 2019].

47 Thanks to the development of the next generation sequencing (NGS) technologies, and their 48 rapidly decreasing costs, it is currently possible to characterize the gut microbiota of a large 49 number of animals. It allows a deeper comprehension of the differences between animals 50 concerning their microbial composition and diversity, which could partially be mediated by

51 the production environment where the animals are raised. Our general aim is to provide

52 further evidence of the effect of different management and environmental factors in the cecal

53 microbial composition and diversity. In relation to this topic, there is a certain amount of 54 information already published. A growing number of studies have revealed changes in rabbit 55 cecal microbial communities exerted by the age [8-Combes et al., 2011] or the type of feed 56 provided to the kits after weaning [6-Zhu et al., 2017; 7-Chen et al., 2019]. One of the 57 important factors of variation is the administration of antibiotics in the feed. In rabbit meat 58 production, antibiotics have been widely administrated to curb mortality, especially after 59 weaning when it often reaches peaks of over $20 \%$ as a result of the onset of gastrointestinal 60 symptoms [9-Gidenne et al., 2010]. Multiple studies have shown alterations caused on gut 61 microbiota by the administration of antibiotics in the feed [5-Zou et al., 2016; 10-Eshar \& 62 Weese, 2014]. Despite the European Union banned their use as growth promoters in animal 63 feeds since 2006 (EC 1831/2003), the administration of one type of antibiotic molecule is 64 still allowed to prevent or treat the emergence of potential infectious diseases on farms. 65 However, substantial efforts are being made towards searching for efficient alternatives 66 which allow a complete withdrawal of antibiotic in animal feeds. In this context, application 
67 of feed restriction during the growing period was proposed as an interesting alternative to the

68 use of antibiotics. Gidenne et al. (2009) [11-Gidenne et al., 2009] demonstrated that feed

69 restriction, despite penalizing animal growth, improves feed efficiency and reduces mortality

70 due to enteric disorders. It is hypothesized that these positive effects could be partially

71 explained by changes in gut microbial composition or activity originated by the application

72 of feed restriction although techniques used so far to study this possible association have not

73 found evidence of it [11-Gidenne et al., 2009].

74

75 Our present study, which comprises a large number of animals in an experimental design

76 involving different management and environmental factors, is intended to unravel changes

77 in diversity and composition of rabbit cecal microbial communities associated with these

78 factors. It will allow a better understanding of how the housing conditions associated with

79 the farm where the animal was raised, the presence of antibiotics in the feed, and feed

80 restriction shape the cecal microbiota of growing rabbits.

82 Results

83 Sequence processing

84 After the removal of doubletons and samples with low sequence counts, 425 rabbit cecal

85 samples (Additional file 1) were represented on 14,928,203 sequence counts clustered into

86963 different OTUs. Each sample had on average 35,125 final sequences (range: 10,157-

87 678,798) and 677 OTUs (range: 197-841) (Additional files 2 and 3). Figure 1 shows two

88 histograms representing the sample richness and the proportion of OTUs presence across 
91

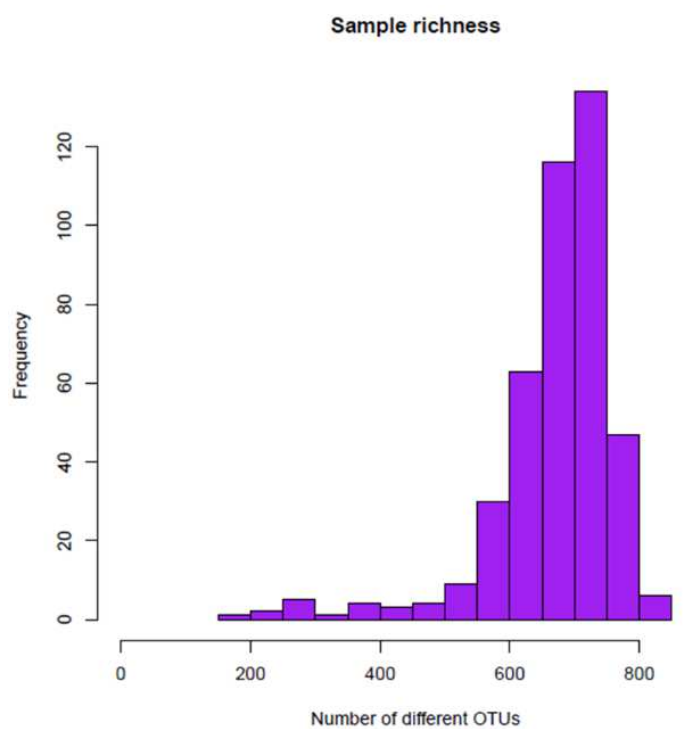

samples. Most of the samples had more than 700 different OTUs (mode $=748$ ) and nearly 140 OTUs were present in all the samples.

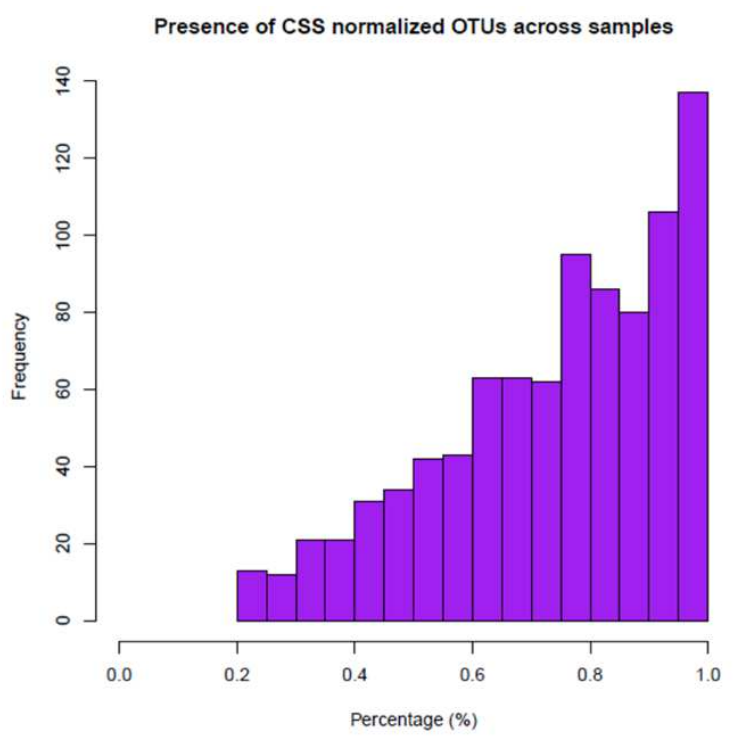

93

94

95 Taxonomic assignment of representative OTUs against the Greengenes reference database 96

97

98

99

100 Figure 1 Sample richness and presence of CSS-normalized OTUs across samples.

(1)

gg_13_5_otus (Additional file 4) revealed the presence of 8 different known phyla with an average of 8 phyla per sample (range: 7-8) (Additional file 5) and 28 different known genera with an average of 24 genera per sample (range: 17-28) (Additional file 6).

\section{Animal management and farm environment shaping cecal microbial alpha diversity}

101 The study of alpha diversity was performed after rarefying the prefiltered and unnormalized 102 OTU table to 10,000 sequences per sample. Rarefaction generated a table which contained 
103 the sequence counts of 963 different OTUs for 425 samples. The average (standard deviation) 104 number of observed OTUs within animal was 560.52 (75.03) and the average Shannon index 105 within animal was 5.09 (0.26). The comparison of alpha diversities revealed that the group 106 of animals raised in farm B had greater alpha diversity than the group of animals raised in 107 farm A (estimated differences of 40.20 (9.83) observed OTUs and 0.17 (0.03) Shannon index; $\left.108 P_{F D R}<0.001\right)$. Furthermore, larger variability in both indexes was observed in farm A than 109 in farm B. No significant differences for the two alpha diversity indexes were found between 110 feeding regimes within both farms (Figure $1, P_{F D R}>0.05$ ), nor between the presence and the 111 absence of antibiotics in the feed within farm B (Figure 1, $P_{F D R}>0.05$ ). 


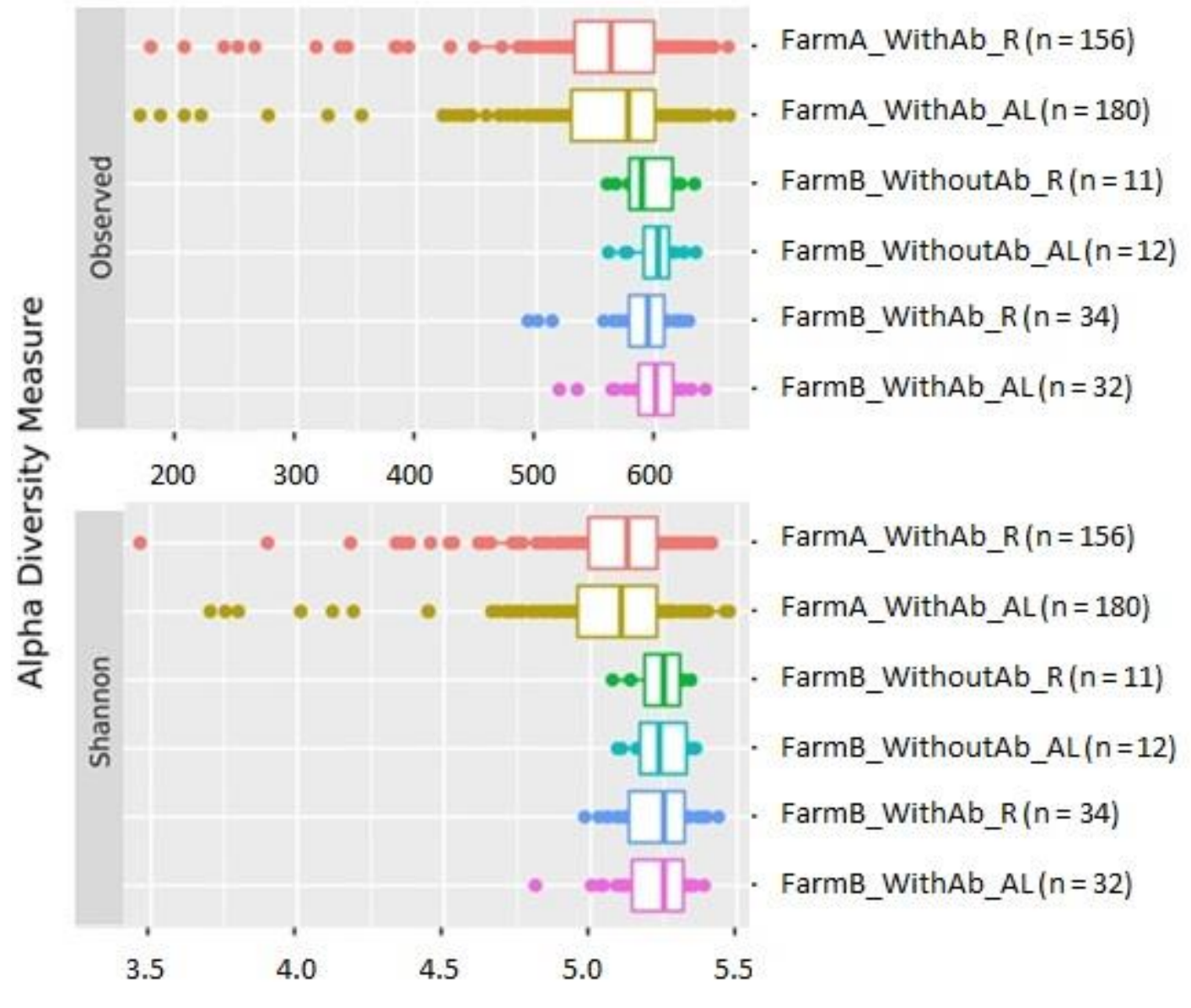

114 Figure 2 Microbial richness and diversity between samples grouped according to

115 management that animals received. The cecal microbial richness and diversity were 116 estimated by the observed number of different OTUs and the Shannon indexes, respectively.

\section{Animal management and farm environment shaping cecal microbial composition}

119 According to the taxonomic assignment of representative sequences (Additional file 4) 120 performed with the UCLUST consensus taxonomy assigner on the Greengenes reference 121 database gg_13_8_99_otus, Firmicutes (76.74\%), Tenericutes (7.22\%) and Bacteroidetes 
$122(6.26 \%)$ were the predominant phyla, accounting for more than $90 \%$ of the microbial 123 diversity, in the rabbit cecal samples studied (Figure 3).

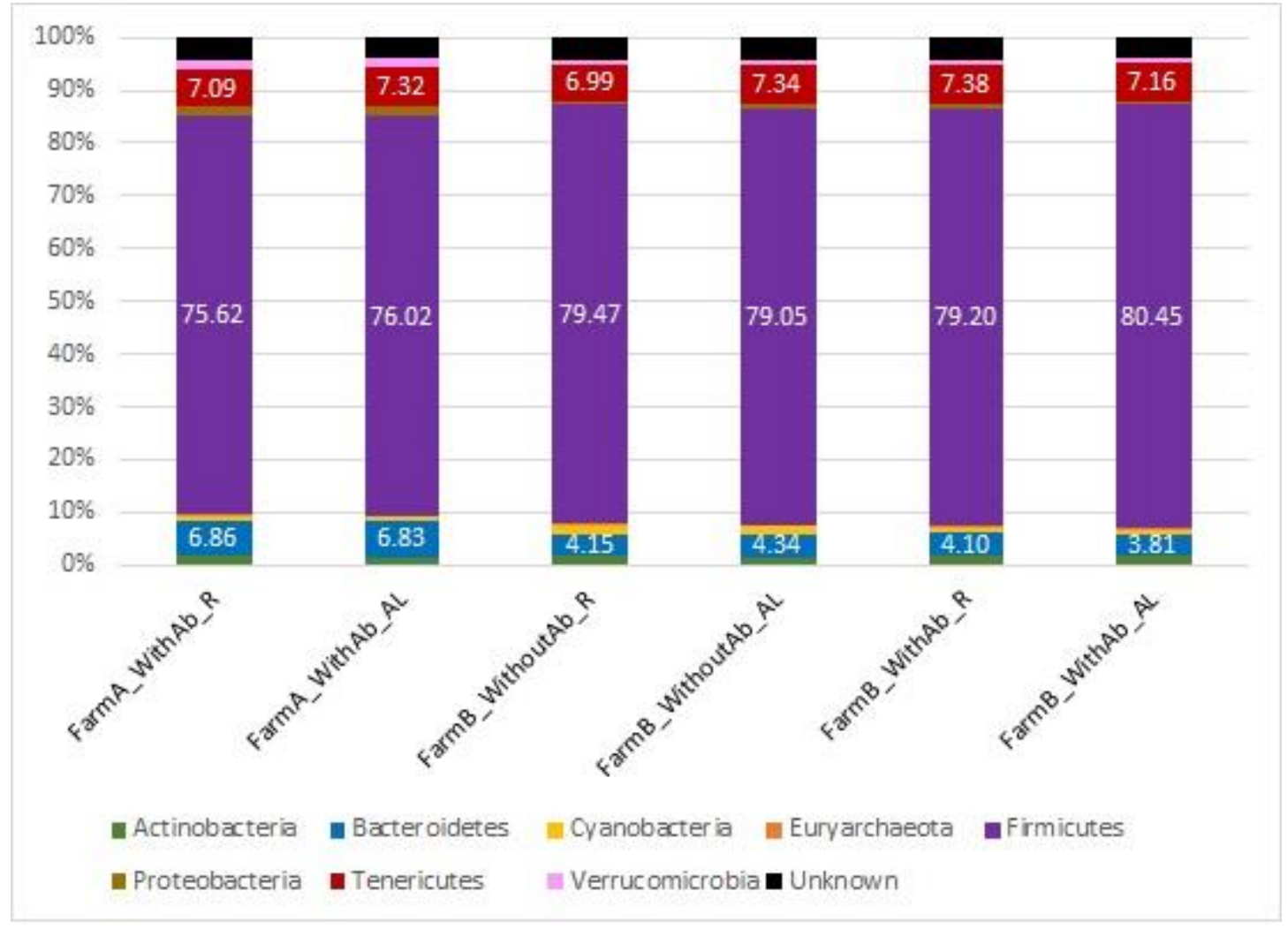

126 Figure 3 Phyla relative abundances of samples grouped according to farm, level of 127 feeding and presence of antibiotics in the feed.

129 Differential cecal microbial composition across farms

130 Cecal samples of rabbits raised in farm A showed an overrepresentation of phyla 131 Bacteroidetes, Proteobacteria and Verrucomicrobia while phyla Euryarchaeota, 
132

133

134

135 136 farm.

Cyanobacteria and Firmicutes were found to be overrepresented in cecal samples of rabbits raised in farm B (Table 1).

\section{Table 1 Microbial composition at phylum level in cecal samples of rabbits grouped by}

\begin{tabular}{|c|c|c|c|c|}
\hline Phylum & $\begin{array}{l}\text { Mean relative } \\
\text { abundance in farm } \\
\mathrm{A}(\%)(\mathrm{SD})\end{array}$ & $\begin{array}{l}\text { Mean relative } \\
\text { abundance in farm } \\
\text { B (\%) (SD) }\end{array}$ & $\begin{array}{l}\text { Estimated } \\
\text { difference farm } \\
A-\text { farm } B \pm S E\end{array}$ & $P_{\text {FDR }}$ \\
\hline Actinobacteria & $1.62(0.67)$ & $1.84(0.33)$ & $-0.14 \pm 0.08$ & 0.09 \\
\hline Bacteroidetes & $6.84(1.81)$ & $4.03(0.70)$ & $2.74 \pm 0.22$ & 0.00 \\
\hline Cyanobacteria & $0.77(0.40)$ & $1.05(0.36)$ & $-0.39 \pm 0.05$ & 0.00 \\
\hline Euryarchaeota & $0.13(0.19)$ & $0.44(0.17)$ & $-0.28 \pm 0.02$ & 0.00 \\
\hline Firmicutes & $75.83(3.34)$ & $79.66(1.53)$ & $-3.78 \pm 0.41$ & 0.00 \\
\hline Proteobacteria & $1.83(0.62)$ & $0.66(0.12)$ & $1.14 \pm 0.07$ & 0.00 \\
\hline Tenericutes & $7.21(1.47)$ & $7.25(0.93)$ & $0.00 \pm 0.18$ & 0.99 \\
\hline Verrucomicrobia & $1.62(0.45)$ & $0.91(0.24)$ & $0.68 \pm 0.05$ & 0.00 \\
\hline
\end{tabular}

137

138 Genera Ruminococcus (4.32\%), Blautia (2.96\%) and Oscillospira (2.37\%) dominate meat

139 rabbit cecal microbiota. Most of the relative abundance differences at genus level were found

140 differentially represented between animals raised in the different farms: genera Bacteroides,

141 Parabacteroides, Rikenella, Anaerofustis, Anaerostipes, Clostridium, Coprobacillus,

142 Anaeroplasma and Akkermansia were overrepresented in cecal samples of rabbits raised in

143 farm A while genera Adlercreutzia, Butyricimonas, Odoribacter, Methanobrevibacter,

144 Blautia, Butyrivibrio, Coprococcus, Dehalobacterium, Dorea, Oscillospira, rc4-4 and

145 Oxalabacter were overrepresented in cecal samples of rabbits raised in farm B. Interestingly,

146 genera Epulopiscium, p-75-a5, Phascolarctobacterium, Campylobacter and Desulfovibrio

147 were only found in samples collected from farm A (Table 2). 
Table 2 Relative abundances of genera, grouped by phylum, differentially represented

150 between farms $\left(P_{F D R}<\mathbf{0 . 0 5}\right)$.

\begin{tabular}{|c|c|c|c|}
\hline Genus & $\begin{array}{l}\text { Mean relative } \\
\text { abundance in } \\
\text { farm A }(\%)(\mathrm{SD})\end{array}$ & $\begin{array}{l}\text { Mean relative } \\
\text { abundance in } \\
\text { farm B }(\%)(\mathrm{SD})\end{array}$ & $\begin{array}{l}\text { Estimated } \\
\text { difference farm } \\
A-\text { farm B } \pm \text { SE } \\
\end{array}$ \\
\hline \multicolumn{4}{|l|}{ Actinobacteria } \\
\hline Adlercreutzia & $0.89(0.47)$ & $1.14(0.23)$ & $-0.19 \pm 0.06$ \\
\hline \multicolumn{4}{|l|}{ Bacteroidetes } \\
\hline Bacteroides & $1.88(0.67)$ & $0.80(0.35)$ & $1.10 \pm 0.08$ \\
\hline Butyricimonas & $0.16(0.19)$ & $0.35(0.17)$ & $-0.19 \pm 0.02$ \\
\hline Odoribacter & $0.23(0.21)$ & $0.44(0.20)$ & $-0.21 \pm 0.03$ \\
\hline Parabacteroides & $0.25(0.18)$ & $0.07(0.07)$ & $0.18 \pm 0.02$ \\
\hline Rikenella & $0.39(0.24)$ & $0.18(0.13)$ & $0.25 \pm 0.03$ \\
\hline \multicolumn{4}{|l|}{ Euryarchaeota } \\
\hline Methanobrevibacter & $0.13(0.19)$ & $0.44(0.17)$ & $-0.28 \pm 0.02$ \\
\hline \multicolumn{4}{|l|}{ Firmicutes } \\
\hline Anaerofustis & $0.12(0.08)$ & $0.08(0.04)$ & $0.03 \pm 0.01$ \\
\hline Anaerostipes & $0.17(0.08)$ & $0.12(0.04)$ & $0.06 \pm 0.01$ \\
\hline Blautia & $2.86(0.67)$ & $3.22(0.46)$ & $-0.36 \pm 0.08$ \\
\hline Butyrivibrio & $0.10(0.07)$ & $0.13(0.06)$ & $-0.03 \pm 0.01$ \\
\hline Clostridium & $1.09(0.26)$ & $0.87(0.13)$ & $0.21 \pm 0.03$ \\
\hline Coprobacillus & $0.20(0.27)$ & $0.14(0.08)$ & $0.08 \pm 0.03$ \\
\hline Coprococcus & $1.96(0.42)$ & $2.26(0.29)$ & $-0.28 \pm 0.05$ \\
\hline Dehalobacterium & $0.05(0.08)$ & $0.18(0.03)$ & $-0.13 \pm 0.01$ \\
\hline Dorea & $0.46(0.12)$ & $0.51(0.09)$ & $-0.05 \pm 0.02$ \\
\hline Epulopiscium & $0.14(0.11)$ & $0.00(0.00)$ & $0.15 \pm 0.01$ \\
\hline Oscillospira & $2.11(0.53)$ & $2.85(0.31)$ & $-0.79 \pm 0.07$ \\
\hline$p-75-a 5$ & $0.13(0.06)$ & $0.00(0.00)$ & $0.13 \pm 0.01$ \\
\hline Phascolarctobacterium & $0.27(0.24)$ & $0.00(0.00)$ & $0.26 \pm 0.03$ \\
\hline$r c 4-4$ & $0.13(0.06)$ & $0.23(0.03)$ & $-0.10 \pm 0.01$ \\
\hline \multicolumn{4}{|l|}{ Proteobacteria } \\
\hline Campylobacter & $0.08(0.08)$ & $0.00(0.00)$ & $0.08 \pm 0.01$ \\
\hline Desulfovibrio & $0.58(0.22)$ & $0.00(0.00)$ & $0.57 \pm 0.03$ \\
\hline Oxalabacter & $0.10(0.06)$ & $0.13(0.03)$ & $-0.03 \pm 0.01$ \\
\hline \multicolumn{4}{|l|}{ Tenericutes } \\
\hline Anaeroplasma & $0.23(0.18)$ & $0.10(0.09)$ & $0.12 \pm 0.02$ \\
\hline \multicolumn{4}{|l|}{ Verrucomicrobia } \\
\hline Akkermansia & $1.62(0.45)$ & $0.91(0.23)$ & $0.68 \pm 0.05$ \\
\hline
\end{tabular}


152 The analyses on the CSS-normalized OTUs revealed that 648 out of the 946 OTUs showed

153 signatures significantly different between farms. Out of these, 276 were overrepresented in 154 farm A, while 372 were overrepresented in farm B. Table S1 shows the estimated difference 155 between farms for these OTUs, their sequences and their assignment at the lowest taxonomic 156 level. Only 9 of them could be assigned at species level and 129 were assigned to known 157 genera. These results show remarkable coincidences with those obtained from the analyses 158 directly performed on the relative abundance of taxa at phylum and genera levels. An 159 example that illustrates this match is the overrepresentation of genus Akkermansia in farm 160 A. This genus is encompassed by phylum Verrucomicrobia that is also overrepresented in 161 rabbits raised in farm A, as well as 6 out of the 7 OTUs assigned to this phylum.

Differential cecal microbial composition across feeding regime

164 An overrepresentation of phyla Cyanobacteria (estimated difference R - AL $=0.11 \pm 0.04$;

$\left.165 P_{F D R}=0.04\right)$ and Verrucomicrobia (estimated difference $\left.\mathrm{R}-\mathrm{AL}=0.11 \pm 0.05 ; P_{F D R}=0.04\right)$

166 was found in cecal samples of rabbits fed $\mathrm{R}$ and raised in farm A. On the other hand, phylum

167 Euryarchaeota was overrepresented in animals fed R and raised in farm B (estimated 168 difference $\left.\mathrm{R}-\mathrm{AL}=0.14 \pm 0.04 ; P_{F D R}<0.001\right)$. At genus level, the only significant contrast 169 was observed for $r c 4-4$ which resulted overrepresented in samples from animals fed AL in 170 farm A (estimated difference $\left.\mathrm{R}-\mathrm{AL}=-0.03 \pm 0.01 ; P_{F D R}<0.001\right)$ while in farm B none of 171 the genera resulted differentially represented $\left(P_{F D R}>0.05\right)$ between feeding regimes. The 172 contrasts based on the CSS-normalized OTUs revealed 51 and 9 OTUs differentially 
173 represented between feeding regimes within farms A and B, respectively. Within farm A, 32

174 OTUs were overrepresented in cecal samples of rabbits that were fed AL and 19 OTUs in the

175 samples from rabbits fed R. Within farm B, 7 OTUs were overrepresented in cecal samples

176 of rabbits that were fed AL and 2 OTUs were overrepresented in rabbits that were fed R.

177 Table $\mathrm{S} 2$ shows the estimated difference between feeding regime within farm of these OTUs,

178 their sequences and their assignment at the lowest taxonomic level. The analyses based on 179 the CSS-normalized OTUs within farm A are in full accordance with the analyses performed 180 at genus level given that all OTUs assigned to genus rc4-4 (phylum Firmicutes) were 181 overrepresented in cecal samples of rabbits fed AL.

184 The effect of the presence of antibiotics in the feed could only be assessed within farm B 185 given that all rabbits raised in farm A received feed supplemented with antibiotics. Cecal 186 samples of rabbits that received feed free of antibiotics showed an overrepresentation of 187 phyla Cyanobacteria compared to those that received feed supplemented with antibiotics 188 (estimated difference withoutAb - withAb $=0.49 \pm 0.09 ; P_{F D R}<0.001$ ). In addition, the 189 analyses on the CSS-normalized OTUs revealed an overrepresentation of 15 and 29 OTUs in 190 cecal samples of rabbits that received a feed supplemented or free of antibiotics; respectively. 191 Table S3 shows the estimated difference between the presence and the absence of antibiotics 192 in the feed for the OTUs in which the differences reached the significance threshold. The 193 OTU sequences as well as their assignment at the lowest taxonomic level are also shown in 194 Table S3. Only 1 of these OTUs could bet assigned at species level (Bacteroides fragilis) and 1952 OTUs at genus level (Oscillospira and Coprococcus). 
197 Microbial information as a classifier of cecal samples according to farm environment 198 and animal management

199 Sparse partial least squares-discriminant analyses (sPLS-DA) on the CSS-normalized OTUs 200 were conducted to discriminate samples according to the factors considered in this study (i.e., 201 the farm where the animal was raised, the presence or the absence of antibiotics in the feed 202 and the feeding regime). The tuning process of the sPLS-DA conducted to discriminate 203 samples according to the farm where the rabbits were raised selected 5 OTUs for component 2041 and 1 OTU for component 2 (Figure 4). Component 1 explained $7.00 \%$ of the total variance 205 while component 2 explained $0.67 \%$. The classification performance of this sPLS-DA can 206 be said to be perfect since its overall and balanced error rate (BER) per class across 1000 207 replicates of 5-folds cross-validation runs was $0.00(0.00)$. Furthermore, two OTUs of 208 component 1 had a stability higher than 0.9. 


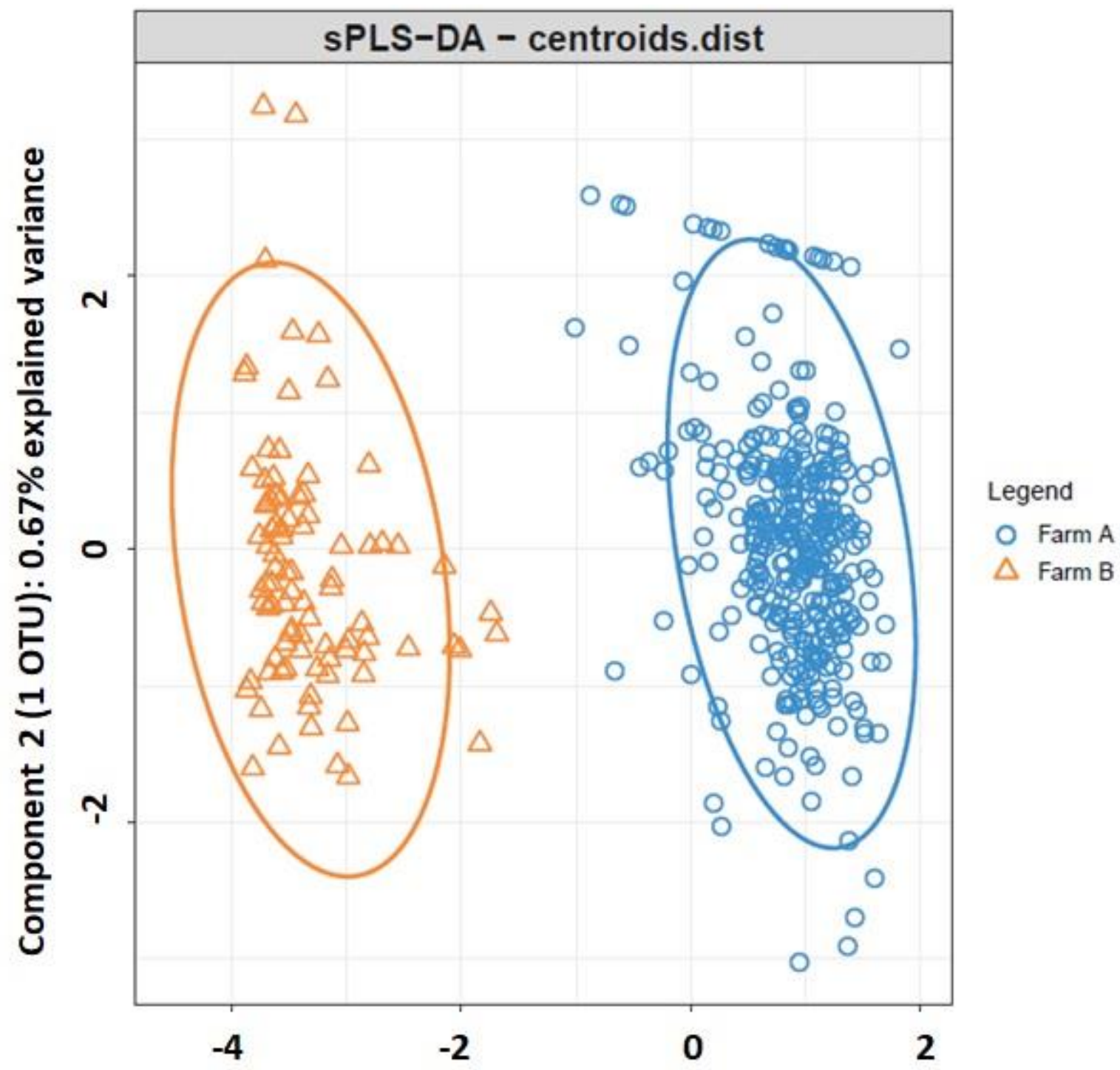

Component 1 (5 OTUs): $7.00 \%$ explained variance

Figure 4 Sparse partial least squares discriminant analysis representing cecal samples of rabbits raised in farm $A$ (blue) and in farm $B$ (orange).

214 The sPLS-DA performed to discriminate samples across feeding regimes within farm A 215 selected 70 OTUs for component 1 and 65 OTUs for component 2 (Figure 5). Component 1 216 explained $2.34 \%$ of the total variance while component 2 explained $5.58 \%$. The cross- 
217 validation assessment of the classification performance of this SPLS-DA showed an overall 218 and BER per class of 0.27 (0.02). The stability of 18 and 5 OTUs selected in components 1 219 and 2, respectively, across the different cross-validation folds was higher than 0.9 .

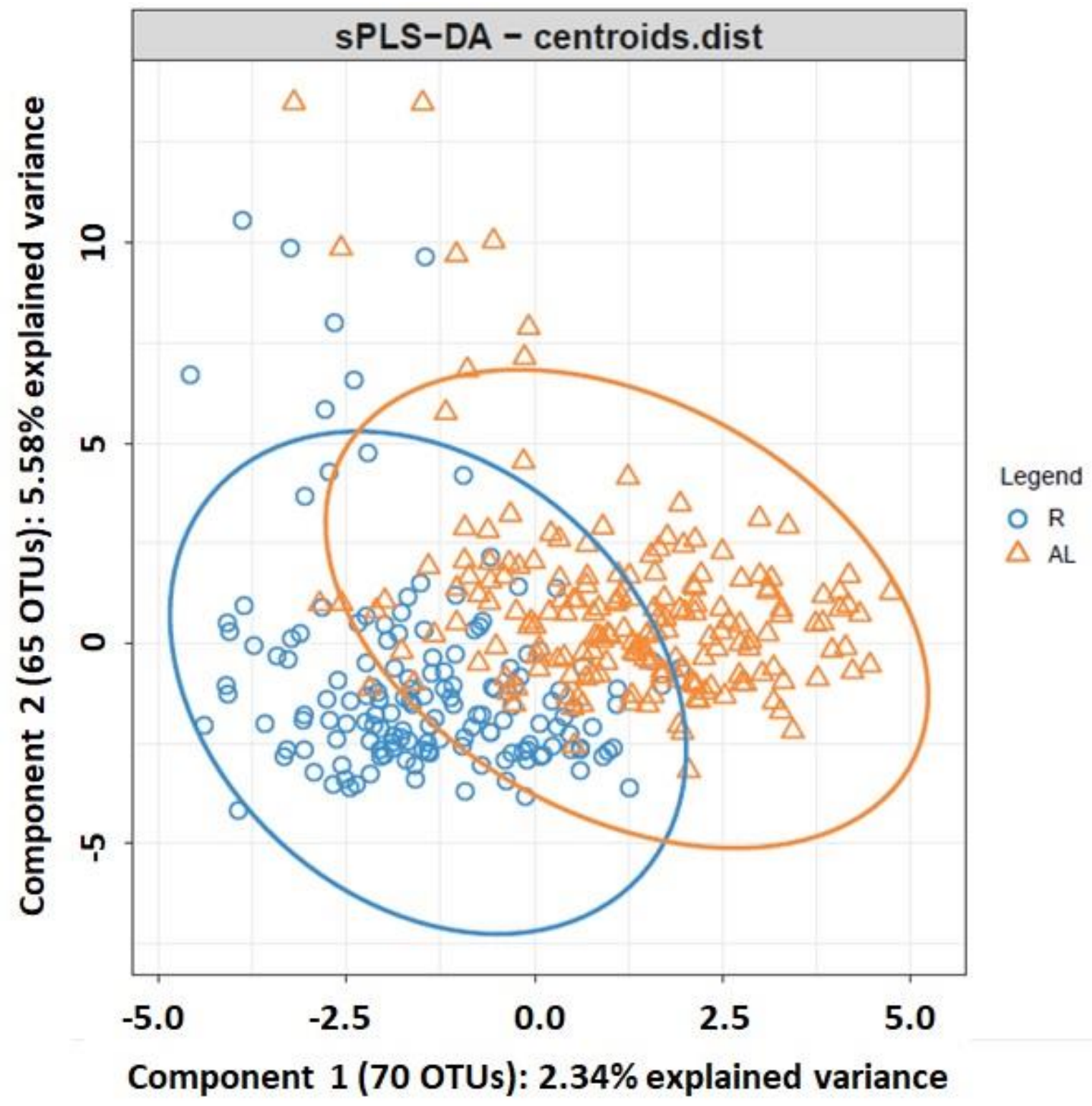
of rabbits raised in farm $A$ and fed $R$ (blue) or AL (orange). 
225 Finally, the sPLS-DA conducted to discriminate samples of animals raised within farm B 226 according to the combination of the presence or not of antibiotics in the feed and the feeding 227 regime, selected 9 OTUs for component 1 and 70 OTUs for component 2 (Figure 6). 228 Component 1 explained $3.05 \%$ of total variance and defined the discrimination between 229 samples from animals fed withAb and those fed withoutAb. On the other hand, component 2302 explained $3.05 \%$ of total variance and defined the discrimination between samples from 231 animals fed $\mathrm{R}$ and those belonging to animals fed AL. The cross-validation assessment of the 232 classification performance of this sPLS-DA showed an overall BER of $0.32(0.15)$. The BER 233 per class was $0.34(0.12)$ for samples fed $\mathrm{R}$ withoutAb, $0.46(0.14)$ for samples fedAL 234 withoutAb, $0.29(0.11)$ for samples fed $\mathrm{R}$ withAb, and $0.20(0.07)$ for samples fed AL 235 withAb. The stability of 3 and 11 OTUs selected in components 1 and 2, respectively, across 236 the different cross-validation folds was higher than 0.9. 


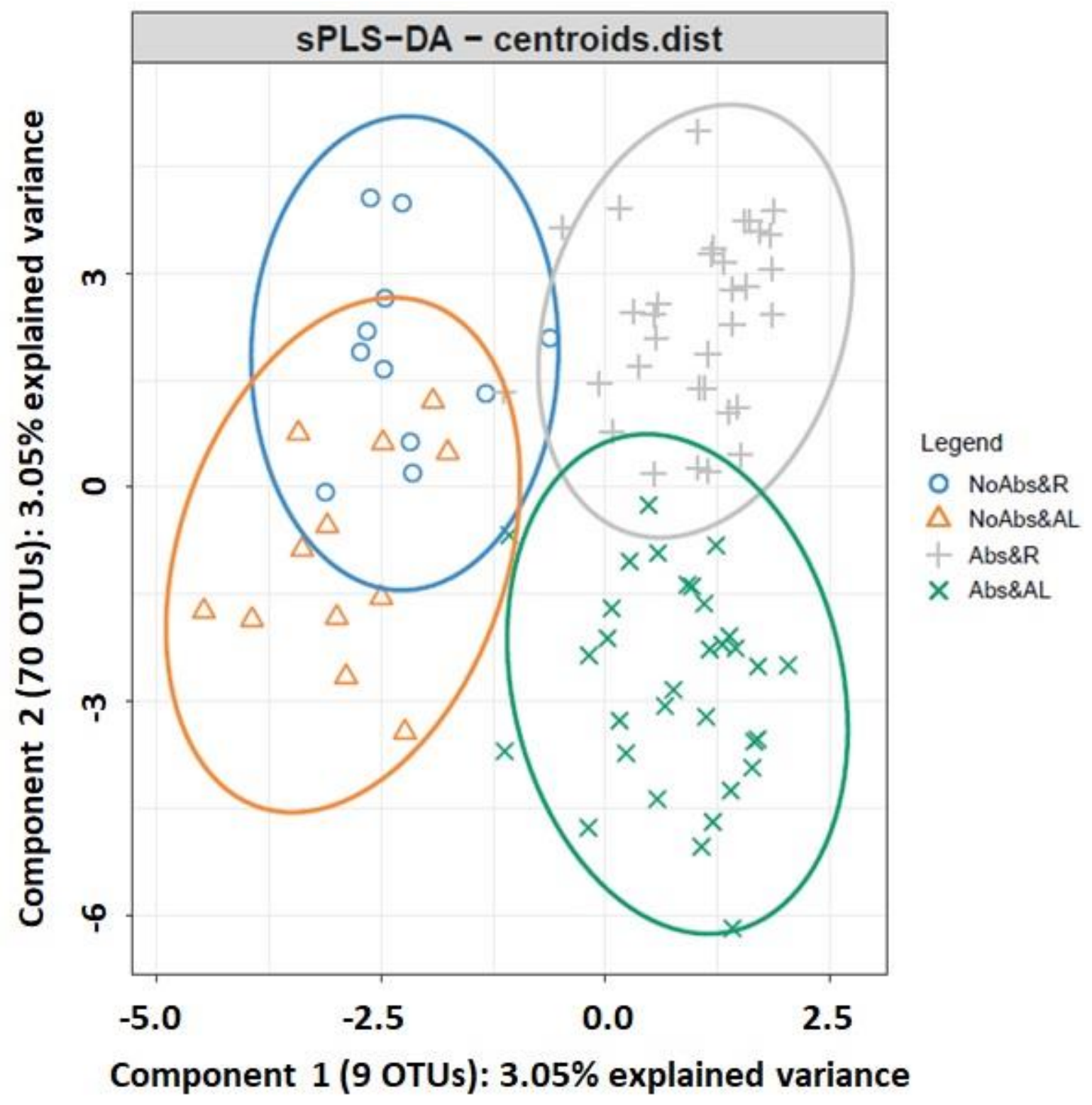

239 Figure 6 Sparse partial least squares discriminant analysis representing cecal samples 240 of rabbits raised in farm $B$ and fed $R$ withoutAb (blue), fed AL withoutAb (orange), fed $241 \quad$ R withAb (gray) and fed AL withAb (green). 


\section{Discussion}

245 The influences of farm environment and common commercial practices of animal 246 management on their gut microbiota are not yet well known in many livestock species. In 247 this study, we have aimed to disentangle potential changes in microbial diversity and 248 composition of meat rabbit cecal communities as a result of being raised in different farms 249 and subjected to different handling during their growing period. To shed light on this matter, 250 we conducted a microbiota comparison of a large number of rabbits raised under different 251 housing conditions, feeding regimes, and fed with feed supplemented or free of antibiotics.

253 The Illumina MiSeq sequence processing of samples collected from these animals revealed 254 that phyla Firmicutes, Tenericutes and Bacteroidetes dominate the growing meat rabbit cecal 255 ecosystem representing more than $90 \%$ of its entire microbial composition. This fact is in 256 accordance with previous studies that have characterized the rabbit cecal microbiota [5-Zou 257 et al., 2016; 7-Chen et al., 2019; 12-Velasco-Galilea et al., 2018] and reported Firmicutes as 258 the predominant phylum. However, there are discrepancies between studies in establishing

259 which other phyla are also prevalent in this ecosystem. Whereas we found phyla Tenericutes 260 and Bacteroidetes representing 7.22 and $5.93 \%$ of the cecal microbial composition, 261 respectively, Chen et al. 2019 [7] and Zou et al. 2016 [5] reported Bacteroidetes as the second 262 predominant phylum representing $18 \%$ and $20 \%$ of New Zealand White and Rex rabbit cecal 263 microbial composition, respectively. Conversely, other studies that have previously 264 characterized meat rabbit fecal microbiota identified phyla Proteobacteria and 265 Verrucomicrobia in higher relative abundances [13-Kylie et al., 2018; 10-Eshar and Weese, 266 2014]. Velasco-Galilea et al. 2018 [12] reported Firmicutes (76.42\%), Tenericutes (7.83) and 
267

268

269

270

271

272

273

274

275

276

277

278

279

280

281

282

283

284

285

286

287

288

289

Bacteroidetes (7.42) as the predominant phyla of meat rabbit fecal and cecal microbial communities. These discrepancies found across studies could be attributed to technical issues (e.g., pair of primers, sequencing platform, bioinformatic pipeline employed to process raw sequences or reference database used for the taxonomic assignment of the representative sequences) or to purely biological reasons (e.g., breed, age or section of the GIT sampled). Nonetheless, Kylie et al. (2018) [13] depicted that the relative increase in less beneficial phyla, such as Proteobacteria, could be related to seasonal climate changes that impact directly to rabbit health. This impact affects the susceptibility to enteritis and possibly feed conversion efficiency. In any case, this phylum is more prevalent in farm A where the animals were more exposed to changes in climate conditions.

Regarding the alpha diversity assessment, Shannon and the observed number of OTUs indexes revealed the existence of significant differences between housing conditions (i.e., the experimental farm where the rabbits were raised). Cecal samples collected from rabbits raised in farm B had greater richness and diversity that those belonging to animals raised in farm A. This could be explained by more stable environmental conditions in farm B (i.e., facility better insulated) than in farm A. This combined with the fact that samples of animals raised in farm A were collected from rabbits produced in 4 different batches, could also explain the larger variability in both indexes observed in this farm [13-Kylie et al., 2018]. Despite not having observed significant differences between the presence or not of antibiotic in the feed, nor between feeding regimes, it is noteworthy to mention that samples collected from animals fed AL in both farms had a greater, although not significant, richness than those fed $\mathrm{R}$. This fact is consistent with previous studies in mice that observed a lower alpha diversity in 
animals with a restricted level of feeding [14-O’Neil et al., 2017; 15-Chen et al., 2016; 16-

291 Zarrinpar et al., 2014]. Surprisingly, but in agreement with our results, studies performed in

292 pigs [17-Soler et al., 2017], chicken [18-Kumar et al., 2018] and Rex rabbits [5] also did not

293 show clear significant differences on alpha diversity indexes between animals fed on diets

294 with antibiotics with respect to those on diets free of antibiotics. Nevertheless, these studies

295 were able to detect differences in the relative abundances of some specific species between

296 diets. For example, Kumar et al. 2018 [18] found that the inclusion of bacitracin in the feed

297 did not affect the chicken bacterial phyla. However, they observed differences between the

298 control and the bacitracin-fed group in the ileal and cecal bacterial populations at lower 299 taxonomic levels.

301 Despite the lack of differences in microbial diversity and richness across management factors 302 (except for the farm); univariate studies revealed differential microbial composition across 303 the studied factors. In addition, the performed multivariate analysis evidenced a certain 304 classification power of the samples on the different levels of management and environment 305 factors based on the microbial composition of the samples.

306 As it might be expected, analyses of variance confirmed that the largest modification of meat 307 rabbit cecal microbial composition is generated by the housing conditions (in this case 308 represented by the farm factor). Our results revealed that the relative abundances of 6 out of 3098 phyla are differentially represented between both farms. At genus level, we detected 310 significant differences in the relative abundances of almost all of them. Genera Bacteroides, 311 Parabacteroides, Rikenella, Anaerofustis, Anaerostipes, Clostridium, Coprobacillus, 312 Anaeroplasma and Akkermansia were enriched in cecal samples of rabbits housed in farm A. 
313 The first three belong to phylum Bacteroidetes and genus Bacteroides is the most abundant

314 of them in meat rabbit cecum. Species of this genus are anaerobic Gram-negative members

315 of the family Bacteroidaceae that play an important role in the degradation of vegetal

316 polysaccharides and amino acid fermentation in the mammal GIT [19-Fang et al.,2017; 20-

317 Dai et al., 2011]. Moreover, this genus is involved in propionic acid and lactate formation

318 depending on nitrogen organic availability. Nonetheless, some authors showed that great

319 amounts of Bacteroides could predict obesity tendency. Parabacteroides is also an anaerobic

320 Gram-negative bacterium (family Porphyromonadaceae) involved in amino acid transport

321 and metabolism, energy production and conversion, lipid transport and metabolism,

322 recombination and repair, cell cycle control, cell division, and cell motility in the intestinal

323 microbiota of the growing rabbit [21-Sun et al., 2020]. This genus was specifically found in

324 the cecal microbiota of mice raised in conventional conditions and absent in those raised in

325 pathogen-free facilities in a study performed under different housing conditions [22- Müller

326 et al., 2016].

327 Within phylum Firmicutes, genus Clostridium (family Clostridiaceae) is an anaerobic Gram-

328 positive bacterium that inhabits the GIT of many mammals where it acts by degrading

329 cellulose. However, some Clostridium species (e.g., C. perfringens and C. difficile) are

330 pathogenic, and an enrichment of this genus has previously been described in rabbits affected

331 by epizootic rabbit enteropathy [23- Bäuerl et al., 2014]. This genus, together with genus

332 Bacteroides, was found enriched in the cecal microbiota of mice housed in open cages

333 compared with those kept in individual ventilated cages [24-Thoene-Reineke et al., 2014].

334 Both genera have been associated with an exacerbation of the intestinal inflammatory

335 response in mammals [25-Terán-Ventura et al., 2010]. Genus Anaerofustis (family 
Eubacteriaceae) has been found enriched in cecal samples of rabbits affected by

337 paratuberculosis infection (Mycobacterium avium) [26-Arrazuria et al., 2016].

338 Within phylum Verrucomicrobia, genus Akkermansia is an anaerobic Gram-negative 339 bacterium that encompasses mucin degrader species [27-Belzer et al., 2012]. In the cecum, a 340 proper enrichment of this genus could maintain a suitable mucosal turn-over, thus exerting a 341 protective effect that could help the animal to deal with inflammatory processes.

342 It is worth mentioning that we have detected genera Epulopiscium, p-75-a5, 343 Phascolarctobacterium, Campylobacter and Desulfovibrio only in the cecal samples of 344 rabbits housed in farm A. The first three are encompassed within phylum Firmicutes. Genus 345 Epulopiscium is a large size Gram-positive bacterium that has a nutritional symbiotic 346 relationship with surgeonfish that eats algae and detritus. This bacterium is physically similar 347 to the phylogenetically related Metabacterium polyspora which is an endospore-producing 348 bacterium isolated from the cecum of guinea pigs [28-Angert et al., 1996]. On the other hand, 349 genera Campylobacter and Desulfovibrio are Gram-negative bacteria that belong to phylum 350 Proteobacteria. Some species of these genera are pathogens responsible for mammal's 351 infections and diarrheas. The exclusive presence of these genera in farm A could indicate the 352 existence of a potential dysbiosis of the animals raised in that facility that could affect their 353 sanitary status and growth. While farm A was a semi-open-air facility, farm B was artificially 354 ventilated and offered more controlled environmental conditions that favor animal growth. 355 Moreover, the presence of sulfate-reducing bacteria (SRB) such as Desulfovibrio could be 356 enhanced by sulfate-secreting bacteria (SSB) such as Rikenella in farm A where this genus 357 is significantly more predominant. It is noteworthy to mention that SRB could also obtain 
sulfate via cross-feeding mediated by Bacteroides-encoded sulfatases [29-Rey et al., 2013],

359 and interestingly, this phylum is more prevalent in farm A.

360 Regarding sample classification based on the sPLS-DA study, given the important 361 differences in gut microbial composition found between farms, a perfect classification of the

362 samples can be achieved with only 5 OTUs. One of these 5 OTUs was overrepresented in 363 farm B and belonged to family S24-7 (phylum Bacteroidetes). The remaining 4 were 364 overrepresented in farm A and belonged to family Barnesiellaceae (phylum Bacteroidetes), 365 order Bacteroidales (phylum Bacteroidetes), and genera Desulfovibrio (phylum 366 Proteobacteria) and Bacteroides (phylum Bacteroidetes). It is worth mentioning that these 5 367 OTUs were also declared as differentially represented between farms by the univariate 368 analyses.

Within farm B, the effect of the presence of antibiotics in the feed was assessed by comparing

371 the microbial cecal composition of rabbits fed withAb with that of some animals that received

372 feed withoutAb. As stated above, we did not detect significant differences in alpha diversity 373 between both groups. Nor univariate studies revealed differences in the relative abundances 374 of genera between diets. However, some significant differences were observed at phylum 375 and OTU levels. An overrepresentation of phylum Cyanobacteria was found in rabbits fed 376 without $\mathrm{Ab}$. The detection of this bacterial phylotype, commonly assigned to photosynthetic 377 activity, in the rabbit cecum could suggest contamination during the GIT sampling. However, 378 Zeng et al. 2015 [30] previously reported its presence in rabbit feces. In the present study, all 379 OTUs taxonomically assigned to phylum Cyanobacteria are as well encompassed in the 380 order YS2. Interestingly, it was demonstrated that this order does not really have 
381 photosynthetic capacity and it is currently classified within candidate phylum

382 Melainabacteria [31-Di Rienzi et al., 2013]. The non-photosynthetic cyanobacteria YS2, now

383 named Gastranaerophilales, is a fermenter gut-associated order present in humans and other

384 animals such as squirrels, where its exact role is unknown but it has the capacity to produce

385 hydrogen, fix nitrogen and synthesize vitamins B and K [31-Di Rienzi et al., 2013; 32-

386 Monchamp et al., 2019; 33-Liu et al., 2020]. Our results, in accordance with Kylie et al. 2018

387 [13], revealed that rabbits fed withoutAb exhibited higher abundances of OTUs assigned to

388 phylum Bacteroidetes than those fed withAb. In addition, samples of rabbits that received

389 antibiotics had a significant increase of an OTU taxonomically assigned to genus

390 Coprococcus. Interestingly, a study that evaluated the differences in bacterial communities

391 of Rex rabbits fed with different antibiotics also found an overrepresentation of this

392 bacterium in animals treated with zinc bacitracin [5-Zou et al., 2016]. Coprococcus is an

393 anaerobic bacterium that may protect against colon cancer in humans by producing butyric

394 acid [34-Ai et al., 2019]. We hypothesized that the administration of antibiotics could

395 modulate the abundance of some Coprococcus species to provide intestinal protection on

396 meat rabbits. However, it is important to recognize that the reduced sample size of the group

397 of rabbits fed withoutAb may have limited the statistical power to detect microbial

398 composition differences associated with this factor.

400 Within this farm, the effect of the feeding regime in microbial composition was also assessed

401 by comparing samples of animals fed R with those fed AL. The main difference found was

402 for phylum Euryarchaeota which was overrepresented in animals fed R in farm B. All

403 Euryarchaeota species found in the rabbit cecum belong to genus Methanobrevibacter that 
encompasses different hydrogenotrophic methane-producing species. Previous studies in

405 humans [35-Shen and Maitin, 2015] and cattle [36-McCabe et al., 2015; 37-McGovern et al.,

406 2017] found an overrepresentation of Methanobrevibacter species in individuals submitted

407 to feed restriction and a negative correlation between the abundance of this bacterium and

408 body mass index. The growth of Methanobrevibacter is supported by fermenters such as

409 Gastranaerophilales and butyrate-producing bacteria such as Anaereostipes via interspecies

410 formate/hydrogen transfer [37-Bui et al., 2019]. A study in mice determined that

411 Methanobrevibacter smithii facilitates Bacteroides thetaiotaomicron capacity to digest

412 glycans resulting in increased production of short-chain fatty acids [38-Samuel and Gordon,

413 2006]. The same study defined $M$. smithii as a "power broker" that regulates polysaccharide

414 fermentation efficiency that influences the fat stores. The lower prevalence of methanogenic

415 archaea in farm A could be explained by the high presence of SRB that outcompetes with

416 methanogens for hydrogen consumption. This fact could favor hydrogen sulfide production

417 and compromise the rabbits' health.

Regarding the sample classification based on the sPLS-DA study conducted within farm B,

420 component 1 and component 2 discriminated between animals that received or not antibiotics

421 in the feed and between feeding regimes, respectively. It is worth mentioning that 8 out of 9

422 OTUs selected in component 1 were also declared as differentially represented between the

423 presence or the absence of antibiotics in the feed by the univariate analyses. Within farm A,

424 an sPLS-DA was also performed to classify samples according to the feeding regime using

425 microbial information. In this case, the discrimination achieved was quite poor as, despite a

426 large number of OTUs were selected as classifier variables in the tuning process, the 
427 classification error rate was high. Nevertheless, bootstrap univariate analyses of variance

428 detected some significant differences at all taxonomic levels analyzed between feeding

429 regimes within farm A. At genus level, $r c 4-4$ was overrepresented in animals fed AL. This

430 genus belongs to phylum Firmicutes and it is known as an obesity-associated bacterium [39-

431 Ziętak et al., 2016] and as a pathogenic candidate identified in mice with multiple sclerosis

432 [40-Gandy et al., 2019]. A potential pro-inflammatory role has been proposed for this genus

433 [40] what could be related to a reduced incidence of enteric disorders when feed restriction

434 is applied. It is worth mentioning that family Peptococcaceae, which encompasses genus

$435 \quad r c 4-4$, is strongly related to total rabbit weight gain from weaning to 12-week old [41-North

436 et al., 2019]. Although in our study this genus was prevalent in animals fed AL, its association

437 with weight gain is not clear since the greater growth exhibited by these animals was

438 consequence of higher feed intake.

440 Different approaches have been applied in this study to evaluate the effect of different

441 environments and management practices, commonly used in rabbit production, in their cecal

442 microbial composition and diversity. Our results confirmed that the most important effect is

443 exerted by the environment provided by the farm where the animals were raised. Those raised

444 in the best insulated facility (farm B) appear to have a microbiota characteristic of healthier

445 animals than those raised in the open-air facility (farm A). It is worth mentioning that the

446 rabbits were housed in cages interspersed with feeding regime. This fact could make possible

447 the exchange of microorganisms between animals of different feeding regimes and therefore

448 have reduced the differences observed between regimes. However, the joint consideration of

44970 OTUs in the sPLS-DA made possible a certain discrimination power of samples according 
450 to the level of feeding received by each animal raised in farm A. It implies the existence of

451 cecal microbiota content patterns characteristic of each regime which could be revealed

452 thanks to the univariate analyses conducted at different taxonomic levels. Similarly, the

453 sPLS-DA performed within farm B also involved the consideration of 70 OTUs to

454 discriminate samples according to the amount of feed consumed. Within this farm, the

455 classification of samples regarding the presence or the absence of antibiotics in the feed

456 needed a smaller number of OTUs than the feeding regime but greater than the farm. This

457 suggests that the effect of the presence of antibiotic in feed is stronger than the feeding level.

458 The implication of the discussed microbial composition and diversity differences originated

459 by the studied management and environmental factors on the animals' performance still

460 needs to be investigated. In future studies the role of specific groups of bacteria in rabbit

461 growth and feed efficiency will be analyzed.

\section{Conclusions}

464 The analysis of a large number of animals from a paternal rabbit line has allowed a deeper 465 comprehension of the role played by different management and environmental factors 466 shaping the composition and diversity of cecal microbial communities. It reveals that the

467 housing conditions offered to the rabbits during their growing play a key role that can result

468 in different microbial alpha diversity and composition of almost all species that inhabit the

469 rabbit GIT. This highlights the importance that a stable and controlled environment could 470 have in the intestinal health and, consequently, in animal performance. It seems clear that the 471 better insulated conditions of farm B favored the presence of a gut microbiota characteristic 
472 of healthier animals. Although the level of feeding and the presence of antibiotics in the feed

473 did not modify the global diversity of cecal microbial communities, these factors can increase

474 or decrease the prevalence of specific bacteria which could lead to a microbial composition

475 potentially beneficial for the animal or, at the other extreme, to an origin of future intestinal 476 dysbiosis.

$478 \quad$ Methods

$479 \quad$ Animals and experimental design

480 All biological samples used in the study were collected from animals of an experiment 481 conducted at the Institute of Agrifood Research and Technology (IRTA) in different periods 482 and involving two different farms. The objective of that experiment was to estimate the effect 483 of the interaction between the genotype and the feeding regime (i.e., the amount of feed 484 provided during fattening) on growth, feed efficiency, carcass characteristics, and health 485 status of the animals [42-Piles and Sánchez, 2019]. For this particular study, 425 meat rabbits 486 from Caldes line [43-Gómez et al., 2002] of that experiment were randomly selected. Most 487 of them (336) were raised in 4 different batches in a semi-open-air facility (farm A). The 488 remaining animals (89) were produced in a single batch in another facility under better 489 controlled environmental conditions (farm B). Rabbits raised in farm A were housed in 490 collective cages containing 8 kits each one while those raised in farm B were housed in cages 491 with 6 kits each one. All animals were raised under the same management conditions and 492 received the same standard pelleted diet. Some of the rabbits raised in farm B received a diet 493 free of antibiotics and the rest received the same diet but supplemented with antibiotics. 
Those raised in farm A received oxytetracycline, valnemulin, and colistin while those in farm

495 B received oxytetracycline, valnemulin and neomycin. During the last fattening week all the 496 animals received an antibiotic free diet. Feed was supplied once per day in a feeder with three 497 places for the 4-5 weeks that the fattening lasted. Water was provided ad libitum during the 498 whole fattening period. The animals were under two different feeding regimes: (1) ad libitum 499 (AL) or (2) restricted (R) to $75 \%$ of the AL feed intake. The amount of feed supplied to the 500 animals under $\mathrm{R}$ feeding regime in a given week for each batch was computed as 0.75 times 501 the average feed intake of kits on AL from the same batch during the previous week, plus $50210 \%$ to account for a feed intake increase as the animal grows. Kits were randomly assigned 503 to one of these two feeding regimes after weaning (32 days of age). They were categorized 504 into two groups according to their size at weaning (big if their body weight was greater than $505700 \mathrm{~g}$ or small otherwise) aiming to obtain homogenous groups regarding animal size within 506 feeding regime. A maximum of two kits of the same litter were assigned to the same cage in 507 order to remove the possible association between cage and maternal effects on animal growth 508 during the fattening period. The distribution of these animals across the different levels of 509 management factors is shown in Table 3.

510 Table 3 Distribution of rabbits in groups according to different management factors.

\begin{tabular}{lllll}
\hline Farm & Batch & Feed & Feeding regime & Number of rabbits \\
\hline A & 1 & With antibiotics & Ad libitum & 27 \\
A & 1 & With antibiotics & Restricted & 30 \\
A & 2 & With antibiotics & Ad libitum & 35 \\
A & 2 & With antibiotics & Restricted & 41 \\
A & 3 & With antibiotics & Ad libitum & 61 \\
A & 3 & With antibiotics & Restricted & 53 \\
A & 4 & With antibiotics & Ad libitum & 57 \\
\hline
\end{tabular}




\begin{tabular}{lllll}
\hline A & 4 & With antibiotics & Restricted & 32 \\
B & 5 & With antibiotics & Ad libitum & 32 \\
B & 5 & With antibiotics & Restricted & 34 \\
B & 5 & Without antibiotics & Ad libitum & 12 \\
B & 5 & Without antibiotics & Restricted & 11 \\
\hline
\end{tabular}

511

512 Sample processing, DNA extraction and sequencing

513 Animals were slaughtered (at 66 and 60 days of age in farm A and farm B, respectively) and

514 cecal samples of each rabbit were collected in a sterile tube, kept cold in the laboratory $\left(4^{\circ} \mathrm{C}\right)$

515 and stored at $-80^{\circ} \mathrm{C}$. DNA extraction, amplification, Illumina library preparation and

516 sequencing followed methods described previously [12-Velasco-Galilea et al., 2018]. Whole

517 genomic DNA was extracted from $250 \mathrm{mg}$ of each cecal samples using ZR Soil Microbe

518 DNA MiniPrep ${ }^{\mathrm{TM}}$ kit (ZymoResearch, Freiburg, Germany) according to manufacturer's

519 instructions with the following modification: cecal samples were mechanically lysed in a

520 FastPrep-24 ${ }^{\mathrm{TM}}$ Homogenizer (MP Biomedicals, LLC, Santa Ana, CA, United States) at a

521 speed of $1 \times 6 \mathrm{~m} / \mathrm{s}$ for $60 \mathrm{~s}$ allowing an efficient lysis of archaea and bacteria species. Integrity

522 and purity of DNA extracts were measured with a Nanodrop ND-1000 spectrophotometer

523 equipment (NanoDrop products; Wilmington, DE, United States) according to Desjardins

524 and Conklin's protocol [44- Desjardins and Conklin, 2010]. All DNA extracts had adequate

525 integrity and purity (absorbance ratio $260 \mathrm{~nm} / 280 \mathrm{~nm}>1.6$ ) to avoid PCR inhibition issues.

527 A fragment of the 16S rRNA gene including the V4-V5 hypervariable regions was amplified

528 with F515Y/R926 primer combination (5'-GTGYCAGCMGCCGCGGTAA-3', 5'-

529 CCGYCAATTYMTTTRAGTTT-3') [45-Parada et al., 2016] and then re-amplified in a 
530 limited-cycle PCR reaction to add sequencing adaptors and 8 nucleotide dual-indexed 531 barcodes of multiplex Nextera ${ }^{\circledR}$ XT kit (Illumina, Inc., San Diego CA, United States) 532 following manufacturer's instructions. The initial PCR reactions were performed for each 533 sample using $12.5 \mu 1$ 2x KAPA HiFi HotStart Ready Mix, $5 \mu 1$ forward primer, $5 \mu 1$ reverse 534 primer and $2.5 \mu \mathrm{l}$ template DNA $(5 \mathrm{ng} / \mu \mathrm{l})$. The initial PCR conditions were as follows: initial 535 denaturation for 3 minutes at $95^{\circ} \mathrm{C}, 25$ cycles of 30 seconds at $95^{\circ} \mathrm{C}, 30$ seconds at $55^{\circ} \mathrm{C}$ 536 and 30 seconds at $72{ }^{\circ} \mathrm{C}$; and final extension for 2 minutes at $72{ }^{\circ} \mathrm{C}$. The addition of indexes 537 and sequencing adaptors to both ends of the amplified regions took place in a second PCR 538 by using $25 \mu 1$ 2x KAPA HiFi HotStart Ready Mix, $5 \mu 1$ index i7, $5 \mu 1$ index i5, $10 \mu 1$ PCR 539 Grade water and $5 \mu$ l concentrated amplicons of initial PCR. The second PCR conditions 540 were as follows: initial denaturation for 3 minutes at $95^{\circ} \mathrm{C}, 8$ cycles of 30 seconds at $95^{\circ} \mathrm{C}$, 54130 seconds at $55^{\circ} \mathrm{C}$ and 30 seconds at $72{ }^{\circ} \mathrm{C}$; and final extension for 5 minutes at $72{ }^{\circ} \mathrm{C}$. Final

542 libraries were cleaned up with AMPure XP beads, validated by running $1 \mu 1$ of a 1:50 dilution 543 on a Bioanalyzer DNA 1000 chip (Agilent Technologies, Inc., Santa Clara, CA, United

544 States) to verify their size, quantified by fluorometry with PicoGreen dsDNA quantification 545 kit (Invitrogen, Life Technologies, Carlsbad, CA, United States), pooled at equimolar 546 concentrations and paired-end sequenced in 5 parallel plates in a Illumina MiSeq 2 x 250 547 platform at the Genomics and Bioinformatics Service (SGB) of the Autonomous University 548 of Barcelona (UAB).

\section{$550 \quad$ Bioinformatic pipeline for OTU calling}

551 Sequence processing was performed using QIIME software (version 1.9.0) [46- Caporaso et 552 al., 2010]. In a first step, the resulting paired-ended V4-V5 16S rRNA gene reads were 
553 assembled into contigs with the python script multiple_join_paired_ends.py. Then the

554 contigs were curated using the script split_libraries.py with default parameters in order to 555 assign them to samples and to discard those with a low-quality (Q19 was the minimum

556 acceptable quality score). Chimeric sequences generated during the process of DNA 557 amplification were detected with UCHIME algorithm [47- Edgar et al., 2011] and removed.

558 The totality of filtered contigs were clustered into operational taxonomic units (OTUs) with 559 a 97\% similarity threshold using the script pick_open_reference_otus.py with default 560 parameters [48- Rideout et al., 2014] that grouped, through UCLUST algorithm [49- Edgar, 561 2010], the sequences against Greengenes reference database (version gg_13_5_otus) and also 562 made a de novo clustering of those that did not match the database. The generated OTU table 563 was filtered at: (1) sample level: by discarding samples with less than 5,000 final sequence 564 counts and at (2) OTU level: by removing the doubleton ones. The filtered OTU table 565 contained the sequence counts of 963 OTUs for 425 samples. Taxonomic assignment of 566 representative sequences of each OTU defined (963) was conducted by mapping them to the

567 Greengenes reference database gg_13_5_otus with the UCLUST consensus taxonomy 568 assigner (QIIME default parameters). The raw sequence data were deposited in the sequence 569 read archive of NCBI under the BioProject accession number PRJNA524130. Metadata, the 570 prefiltered and normalized OTU tables, and corresponding taxonomic classifications are also 571 included as Additional files 1, 2, 3 and 4, respectively.

$573 \quad$ Models and statistical methods

574 In order to study differences in diversity and richness between rabbits grouped according to 575 the farm environment and the management that they received, two alpha diversity indexes 
576 (Shannon and the observed number of OTUs) were computed from the OTU table rarified to

57710,000 sequences per sample with "phyloseq" R package [50-phyloseq]. The statistical

578 method chosen to assess alpha diversity differences between these groups of animals was an

579 analysis of variance that included a factor resulting from the combination of four factors (the

580 farm where the animal was raised, the batch, the presence or the absence of antibiotics in the

581 feed and the feeding regime). The significance threshold was set at 0.05 type I error.

583 Different approaches were considered to assess the influence of the environments and 584 management factors on microbial composition. A bootstrap analysis of variance was 585 individually implemented for each OTU to test whether it was differentially represented 586 between the different categories of the factors studied. This univariate analysis was 587 conducted by normalizing the OTU table with the cumulative sum scaling (CSS) method [51588 Paulson et al., 2013] and only for those OTUs which were detected in at least 5\% of the 589 samples and had a sum of its counts resulting in a frequency greater than $0.01 \%$ of the total 590 sum of all OTUs counts across all samples. It was implemented by fitting a model defined 591 by the combination of the four aforementioned factors by using $\operatorname{lm}()$ function in R [52- R]. 592 Then, the differences between the CSS-normalized OTUs counts in the different levels of the 593 studied factors were tested. The significance between the levels of the main factors: farm, 594 presence of antibiotics in the feed and feeding regime was assessed using an F statistic. When 595 the involved interaction terms were significant, the contrasts of interest were studied nested 596 within the levels of other interacting factors, i.e. feeding regime were studied within farm 597 levels. When the interaction terms were not significant, the effects of the different levels were 598 averaged, i.e. the effects of the levels of the batches within farm A were averaged to present 
599 the effect associated with this farm. In the performed $\mathrm{F}$ tests instead of relying on the

600 theoretical distribution of the statistic under the null hypothesis to define the p-values, they

601 were empirically computed using bootstrap after 1,000 permutations of the dependent

602 variable with respect to the design matrix of factors in the model. The use of bootstrapping

603 enabled the hypothesis test to be done without the need of assuming that data are normally

604 distributed, which is an assumption that fails for OTUs counts. $P$-value was defined as the 605 proportion of bootstrap rounds having an $\mathrm{F}$ statistic value equal or greater than that obtained

606 with the original dataset. $P$-values were corrected defining a false discovery rate (FDR) of 6070.05 [53- Benjamini and Hochberg, 1995]. This bootstrap analysis of variance approach was 608 also implemented to study the effect of the management factors on the relative abundance of 609 bacteria at phylum and genus levels.

611 The value of the microbial information to classify samples into the three factors considered

612 in our study was explored using multivariate techniques. In particular, sparse partial least 613 squares-discriminant analysis (sPLS-DA) [54-Le Cao et al., 2008] was used to find the 614 combination of OTUs that allowed the best classification of cecal samples according to: (1) 615 the farm where the animals were raised, (2) the feeding regime within farm A and (3) the 616 combination of feeding regime and the presence or absence of antibiotics in the feed for the 617 animals raised in farm B. This approach was implemented through the $\mathrm{R}$ package 618 "mixOmics" [55-mixomics]. In a first step, the function tune.splsda() was used to select the 619 optimal sparsity parameters of the sPLS-DA model: the number of components and the 620 number of variables (OTUs) per component. For the tuning process, a 5-fold cross-validation 621 repeated 10 times was performed one component at a time, with a maximum of 4 
622 components, on an input grid of values that indicate the number of variables to select on each

623 component. The sparsity parameters were defined, based on the BER and centroids distance,

624 and then included in the final sPLS-DA model. Samples were represented on the first two

625 components and colored according to their class (e.g., R or AL in the case of the feeding

626 regime) in a sample plot with the function plotIndiv(). The performance of the sPLS-DA

627 model was assessed with a 5-fold cross-validation repeated 1,000 times that randomly split

628 the data in training and validation sets. In this data partition, it was ensured that $20 \%$ of the

629 samples within each level of the discriminant factor were assigned to the validation set. Five

630 different partitions were performed for each replicate to guarantee a different sample

631 distribution in each validation set. The sPLS-DA model with the sparsity parameters

632 previously defined was adjusted in the training set and its classification performance was

633 assessed in the validation set using the overall and BER per class as criteria. The stability of

634 the OTUs selected on each component was also assessed in the cross-validation by computing

635 the selection frequency of each variable across the replicates.

636

637 Additional files

638 Additional file 1: metadata.txt. Metadata associated with the 425 rabbit cecal samples 639 analyzed in this study.

640 Additional file 2: otu_table_prefiltered_unnormalized.txt. Prefiltered and unnormalized 641 OTU table used for statistical analyses in this study.

642 Additional file 3: otu_table_filtered_CSSnormalized.txt. Filtered and CSS-normalized OTU 643 table used for statistical analyses in this study. 
644 Additional file 4: rep_OTUs_tax_assignments.txt. Taxonomic assignments for all OTUs in 645 Additional file 2.

646 Additional file 5: phyla_table.txt. Relative abundances phyla table built from the collapse 647 of the filtered and CSS-normalized OTU table at phylum level.

648 Additional file 6: genera_table.txt. Relative abundances genera table built from the collapse 649 of the filtered and CSS-normalized OTU table at genus level.

650 Additional file 7: Table S1. OTUs differentially represented between farms.

651 Additional file 8: Table S2. OTUs differentially represented between feeding regimes 652 within farms.

653 Additional file 9: Table S3. OTUs differentially represented between the presence and the 654 absence of antibiotics in the feed within farm B.

655

\section{List of abbreviations}

657 AL: ad libitum

658 BER: balanced error rate

659 CSS: cumulative sum scaling

660 FDR: false discovery rate

661 GIT: gastrointestinal tract

662 NGS: next generation sequencing 
663 OTU: operational taxonomic unit

664 PCR: polymerase chain reaction

665 R: restricted

666 SPLS-DA: sparse partial least squares-discriminant analysis

667 SRB: sulfate-reducing bacteria (SRB)

668 SSB: sulfate-secreting bacteria (SRB)

669 WithAb: feed supplemented with antibiotics

670 WithoutAb: feed free of antibiotics

671

672 Declarations

673 Ethics approval and consent to participate

674 This study was carried out in accordance with the recommendations of the animal care and

675 use committee of the Institute for Food and Agriculture Research and Technology

676 (IRTA). The protocol was approved by the committee of the Institute for Food and

677 Agriculture Research and Technology (IRTA).

678

679 Consent for publication

680 Not applicable.

681 
683 The raw sequence data were deposited in the sequence read archive of NCBI under the 684 accession number SRP186982 (BioProject PRJNA524130). Metadata, the prefiltered and 685 unnormalized OTU table, the filtered and CSS-normalized OTU table and corresponding 686 taxonomic assignments have all been included as Additional files 1, 2, 3 and 4, respectively.

687 Relative abundances phyla and genera table have also been included as Additional files 5 and 6886 , respectively. OTUs differentially represented between the studied factors, their sequences 689 and their assignment at the lowest taxonomic level have been included as Additional files 7 , 6908 and 9.

691

\section{Competing interests}

693 The authors declare that they have no competing interests.

694

695 Funding

696 The experimental design of this work was conducted thanks to funding from INIA project 697 RTA2011-00064-00-00. This study was part of the Feed-a-Gene project and received funding 698 from the European Union's H2020 program under grant agreement no. 633531. MVG is a 699 recipient of a "Formación de Personal Investigador (FPI)" pre-doctoral fellowship from 700 INIA, associated with the research project RTA2014-00015-C2-01. YRC was funded by 701 Marie Skłodowska-Curie grant (P-Sphere) agreement no. 6655919 (EU). 
704 JS, MP and OR conceived the experimental design. JS, OR, MP and MVG collected 705 biological samples. MVG, OGR, MP, MG and AS processed the samples in the laboratory. 706 MVG processed and analyzed the sequencing data, interpreted data, prepared figures and 707 tables, and wrote the manuscript. JS and YRC helped analyzing the sequencing data. JS, MG, $708 \mathrm{MP}, \mathrm{MV}$ and YRC helped interpreting the data, and wrote and revised the manuscript. All 709 authors read and approved the final manuscript.

\section{Acknowledgements}

712 We would like to thank Oscar Perucho, Josep Ramon and Carmen Requena (staff of Unitat 713 de Cunicultura, IRTA) for their contribution to data recording and animal care during the 714 experiment. We also acknowledge Nicolas Boulanger and Joana Ribes (Genomics and NGS 715 Unit, CRAG) for their assistance in massive libraries preparation.

717 References

718 [1] Gaskins, H. R. Immunological aspects of host/microbiota interactions at the intestinal 719 epithelium. Gastrointestinal microbiology;1997;2:537-587.

721 [2] Belkaid, Y., Hand, T. W. Role of the microbiota in immunity and inflammation. 722 Cell;2014;157(1):121-141. 
724 [3] Gouet, P. H., Fonty, G. Changes in the digestive microflora of holoxenic* rabbits from 725 birth until adulthood. Annales de biologie animale biochimie biophysique;1979;19:553-566.

726

727 [4] Abecia, L., Fondevila, M., Balcells, J., Lobley, G. E., McEwan, N. R. The effect of 728 medicated diets and level of feeding on caecal microbiota of lactating rabbit does. Journal of 729 applied microbiology;2007;103(4):787-793.

731 [5] Zou, F., Zeng, D., Wen, B., Sun, H., Zhou, Y., Yang, M., et al. Illumina Miseq platform 732 analysis caecum bacterial communities of rex rabbits fed with different antibiotics. AMB 733 express;2016:6(1):100.

734

735 [6] Zhu, Y., Sun, Y., Wang, C., Li, F. Impact of dietary fibre: starch ratio in shaping caecal 736 archaea revealed in rabbits. Journal of animal physiology and animal 737 nutrition;2017;101(4);635-640.

739 [7] Chen, S. Y., Deng, F., Jia, X., Liu, H., Zhang, G. W., Lai, S. J. Gut microbiota profiling 740 with differential tolerance against the reduced dietary fibre level in rabbit. Scientific 741 reports;2019;9(1):1-9. 
743 [8] Combes, S., Michelland, R. J., Monteils, V., Cauquil, L., Soulié, V., Tran, N. U., et al. 744 Postnatal development of the rabbit caecal microbiota composition and activity. FEMS 745 microbiology ecology;2011;77(3):680-689.

746

747 [9] Gidenne, T., García, J., Lebas, F., Licois, D. Nutrition and feeding strategy: interactions 748 with pathology. Nutrition of the rabbit;2010;20:179.

750 [10] Eshar, D., Weese, J. S. Molecular analysis of the microbiota in hard feces from healthy 751 rabbits (Oryctolagus cuniculus) medicated with long term oral meloxicam. BMC veterinary 752 research;2014;10(1):62.

753

754 [11] Gidenne, T., Combes, S., Feugier, A., Jehl, N., Arveux, P., Boisot, P., et al. Feed 755 restriction strategy in the growing rabbit. 2. Impact on digestive health, growth and carcass 756 characteristics. Animal;2009;3(4):509-515.

757

758 [12] Velasco-Galilea, M., Piles, M., Viñas, M., Rafel, O., González-Rodríguez, O., 759 Guivernau, M., et al. Rabbit microbiota changes throughout the intestinal tract. Frontiers in 760 microbiology;2018;9:2144. 
762

763

764

765

766

767

768

769

770

771

772

773

774

775

776

777

778

779

780

781

[13] Kylie, J., Weese, J. S., Turner, P. V. Comparison of the fecal microbiota of domestic commercial meat, laboratory, companion, and shelter rabbits (Oryctolagus cuniculi). BMC veterinary research;2018;14(1):143.

[14] O’Neil, D. S., Stewart, C. J., Chu, D. M., Goodspeed, D. M., Gonzalez-Rodriguez, P. J., Shope, C. D., et al. Conditional postnatal deletion of the neonatal murine hepatic circadian gene, Npas2, alters the gut microbiome following restricted feeding. American journal of obstetrics and gynecology;2017;217(2):218-e1.

[15] Chen, J., Toyomasu, Y., Hayashi, Y., Linden, D. R., Szurszewski, J. H., Nelson, H., et al. Altered gut microbiota in female mice with persistent low body weights following removal of post-weaning chronic dietary restriction. Genome medicine;2016;8(1):103.

[16] Zarrinpar, A., Chaix, A., Yooseph, S., Panda, S. Diet and feeding pattern affect the diurnal dynamics of the gut microbiome. Cell metabolism;2014;20(6):1006-1017.

[17] Soler, C., Goossens, T., Bermejo, A., Migura-García, L., Cusco, A., Francino, O., et al. Digestive microbiota is different in pigs receiving antimicrobials or a feed additive during the nursery period. PloS one;2018;13(5). 
782

783

784

785

786

787

788

789

790

791

792

793

794

795

796

797

798

799

800

801

[18] Kumar, S., Chen, C., Indugu, N., Werlang, G. O., Singh, M., Kim, et al. Effect of antibiotic withdrawal in feed on chicken gut microbial dynamics, immunity, growth performance and prevalence of foodborne pathogens. PLoS One;2018;13(2).

[19] Fang, S., Xiong, X., Su, Y., Huang, L., Chen, C. 16S rRNA gene-based association study identified microbial taxa associated with pork intramuscular fat content in feces and cecum lumen. BMC microbiology;2017;17(1):162.

[20] Dai, Z. L., Wu, G., Zhu, W. Y. Amino acid metabolism in intestinal bacteria: links between gut ecology and host health. Frontiers bioscience;2011;16(1):1768-86.

[21] Sun, X., Shen, J., Liu, C., Li, S., Peng, Y., Chen, C., et al. L-Arginine and Ncarbamoylglutamic acid supplementation enhance young rabbit growth and immunity by regulating intestinal microbial community. Asian-Australasian journal of animal sciences;2020;33(1):166.

[22] Müller, V. M., Zietek, T., Rohm, F., Fiamoncini, J., Lagkouvardos, I., Haller, D., et al. Gut barrier impairment by high-fat diet in mice depends on housing conditions. Molecular nutrition \& food research;2016;60(4):897-908. 
802 [23] Bäuerl, C., Collado, M. C., Zuniga, M., Blas, E., Martínez, G. P. Changes in cecal 803 microbiota and mucosal gene expression revealed new aspects of epizootic rabbit 804 enteropathy. PLoS One;2014;9(8).

805

806 [24] Thoene-Reineke, C., Fischer, A., Friese, C., Briesemeister, D., Göbel, U. B., 807 Kammertoens, T., et al. Composition of intestinal microbiota in immune-deficient mice kept 808 in three different housing conditions. PloS one;2014;9(11).

809

810 [25] Terán-Ventura, E., Roca, M., Martin, M. T., Abarca, M. L., Martinez, V., Vergara, P. 811 Characterization of housing-related spontaneous variations of gut microbiota and expression 812 of toll-like receptors 2 and 4 in rats. Microbial ecology;2010;60(3):691-702.

814 [26] Arrazuria, R., Elguezabal, N., Juste, R. A., Derakhshani, H., Khafipour, E. 815 Mycobacterium avium subspecies paratuberculosis infection modifies gut microbiota under 816 different dietary conditions in a rabbit model. Frontiers in microbiology;2016;7:446.

818 [27] Belzer, C., De Vos, W. M. Microbes inside-from diversity to function: the case of 819 Akkermansia. The ISME journal;2012;6(8):1449-1458. 
821 [28] Angert, E. R., Brooks, A. E., Pace, N. R. Phylogenetic analysis of Metabacterium 822 polyspora: clues to the evolutionary origin of daughter cell production in Epulopiscium 823 species, the largest bacteria. Journal of bacteriology;1996;178(5:1451-1456.

824

825 [29] Rey, F. E., Gonzalez, M. D., Cheng, J., Wu, M., Ahern, P. P., Gordon, J. I. Metabolic 826 niche of a prominent sulfate-reducing human gut bacterium. Proceedings of the national 827 academy of sciences;2013;110(33):13582-13587.

828

829 [30] Zeng, B., Han, S., Wang, P., Wen, B., Jian, W., Guo, W., et al. The bacterial 830 communities associated with fecal types and body weight of rex rabbits. Scientific 831 reports;2015;5:9342-9350.

833 [31] Di Rienzi, S. C., Sharon, I., Wrighton, K. C., Koren, O., Hug, L. A., Thomas, B. C., et 834 al. The human gut and groundwater harbor non-photosynthetic bacteria belonging to a new 835 candidate phylum sibling to Cyanobacteria. Elife;2013;2:e01102.

837 [32] Monchamp, M. E., Spaak, P., Pomati, F. Long term diversity and distribution of non838 photosynthetic cyanobacteria in peri-alpine lakes. Frontiers in microbiology;2019;9:3344. 
840 [33] Liu, P. Y., Cheng, A. C., Huang, S. W., Lu, H. P., Oshida, T., Liu, W., et al. Body-size

841 Scaling is Related to Gut Microbial Diversity, Metabolism and Dietary niche of Arboreal

842 folivorous flying Squirrels. Scientific reports;2020;10(1):1-12.

843

844 [34] Ai, D., Pan, H., Li, X., Gao, Y., Liu, G., Xia, L. C. Identifying gut microbiota associated 845 with colorectal cancer using a zero-inflated lognormal model. Frontiers in 846 microbiology;2019;10:826.

847

848 [35] Shen, Q., Maitin, V. Obesity-associated gut microbiota: characterization and dietary 849 modulation. In Diet-Microbe Interactions in the Gut. Academic press;2015:149-171.

850

851 [36] McCabe, M. S., Cormican, P., Keogh, K., O’Connor, A., O’Hara, E., Palladino, R. A., 852 et al. Illumina MiSeq phylogenetic amplicon sequencing shows a large reduction of an 853 uncharacterised Succinivibrionaceae and an increase of the Methanobrevibacter gottschalkii 854 clade in feed restricted cattle. PloS one;2015;10(7).

855

856 [36] McGovern, E., McCabe, M. S., Cormican, P., Popova, M., Keogh, K., Kelly, A. K., et 857 al. Plane of nutrition affects the phylogenetic diversity and relative abundance of 858 transcriptionally active methanogens in the bovine rumen. Scientific reports;2017;7(1):1-10. 
[37] Bui, T. P. N., Schols, H. A., Jonathan, M., Stams, A. J., de Vos, W. M., Plugge, C. M.

861 Mutual Metabolic Interactions in Co-cultures of the Intestinal Anaerostipes rhamnosivorans

862 With an Acetogen, Methanogen, or Pectin-Degrader Affecting Butyrate Production.

863 Frontiers in microbiology;2019;10:2449.

864

865 [38] Samuel, B. S., Gordon, J. I. A humanized gnotobiotic mouse model of host-archaeal866 bacterial mutualism. Proceedings of the national academy of sciences;2006;103(26):1001186710016.

868

869

[39] Ziętak, M., Kovatcheva-Datchary, P., Markiewicz, L. H., Ståhlman, M., Kozak, L. P.,

870 Bäckhed, F. Altered microbiota contributes to reduced diet-induced obesity upon cold 871 exposure. Cell metabolism;2016;23(6):1216-1223.

872

873 [40] Gandy, K. A. O., Zhang, J., Nagarkatti, P., Nagarkatti, M. The role of gut microbiota in 874 shaping the relapse-remitting and chronic-progressive forms of multiple sclerosis in mouse 875 models. Scientific reports;2019;9(1):1-17.

876

877 [41] North, M. K., Dalle Zotte, A., Hoffman, L. C. Composition of rabbit caecal microbiota 878 and the effects of dietary quercetin supplementation and sex thereupon. World rabbit 879 science;2019;27(4):185-198. 
881 [42] Piles, M., Sánchez, J. P. Use of group records of feed intake to select for feed efficiency

882 in rabbit. Journal of animal breeding and genetics;2019;136(6):474-483.

884 [43] Gómez, E. A., Rafel, O., Ramon, J. The Caldes strain (Spain). Options 885 Méditerranéennes. Série B: Etudes et recherches (CIHEAM);2002.

886

887 [44] Desjardins, P., Conklin, D. NanoDrop microvolume quantitation of nucleic 888 acids. Journal of visualized experiments: JoVE;2010;45.

889

890 [45] Parada, A. E., Needham, D. M., Fuhrman, J. A. Every base matters: assessing small 891 subunit rRNA primers for marine microbiomes with mock communities, time series and 892 global field samples. Environmental microbiology;2016;18(5):1403-1414.

894 [46] Caporaso, J. G., Kuczynski, J., Stombaugh, J., Bittinger, K., Bushman, F. D., Costello, 895 E. K., et al. QIIME allows analysis of high-throughput community sequencing data. Nature 896 methods;2010;7:335-336.

897

898 [47] Edgar, R. C., Haas, B. J., Clemente, J. C., Quince, C., Knight, R. UCHIME improves 899 sensitivity and speed of chimera detection. Bioinformatics;2011;27:2194-2200 
901 [48] Rideout, J. R., He, Y., Navas-Molina, J. A., Walters, W. A., Ursell, L. K., Gibbons,

902 S. M., et al. Subsampled open-reference clustering creates consistent, comprehensive OTU 903 definitions and scales to billions of sequences. PeerJ;2014;2:e545.

904

905 [49] Edgar, R. C. Search and clustering orders of magnitude faster than BLAST. 906 Bioinformatics;2010;26:2460-2461.

907

908 [50] McMurdie, P. J., Holmes, S. phyloseq: an R package for reproducible interactive 909 analysis and graphics of microbiome census data. PloS one;2013;8(4):e61217.

910

911 [51] Paulson, J. N., Stine, O. C., Bravo, H. C., Pop, M. Differential abundance analysis for 912 microbial marker-gene surveys. Nature methods;2013;10(12),1200.

914 [52] R Development Core Team. R: A Language and Environment for Statistical Computing. $9152010[$ http://cran.r-project.org].

917 [53] Benjamini, Y., Hochberg, Y. Controlling the false discovery rate: a practical and 918 powerful approach to multiple testing. Journal of the Royal statistical society: series B 919 (Methodological);1995;57(1):289-300. 
921 [54] Lê Cao, K. A., Rossouw, D., Robert-Granié, C., Besse, P. A sparse PLS for variable 922 selection when integrating omics data. Statistical applications in genetics and molecular 923 biology;2018;7(1).

924

925 [55] Rohart, F., Gautier, B., Singh, A., Le Cao K. A. mixOmics: An R package for 'omics 926 feature selection and multiple data integration. PLoS computational 927 biology;2017;13(11):e1005752. 
Figures
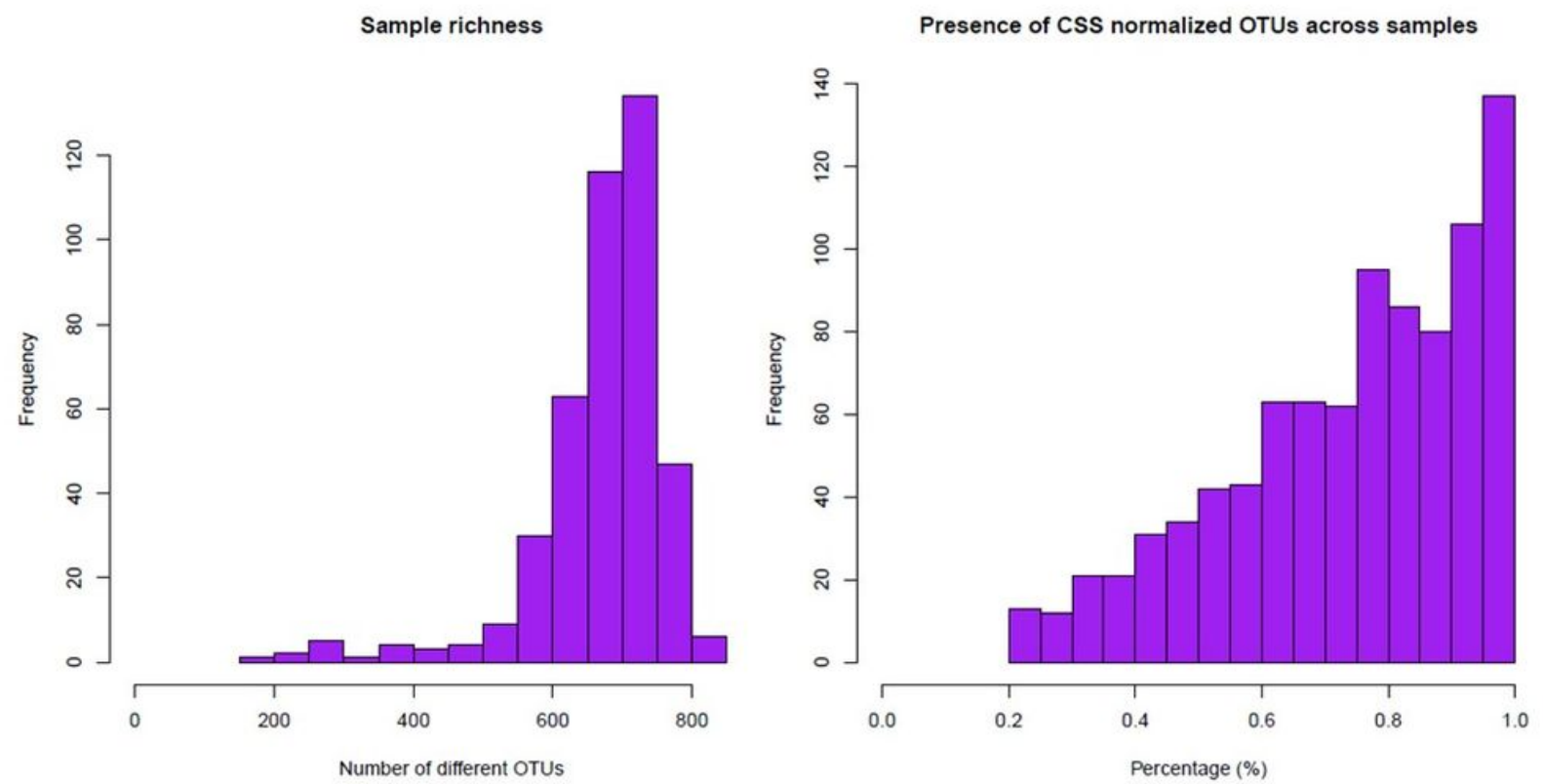

Figure 1

Sample richness and presence of CSS-normalized OTUs across samples. 


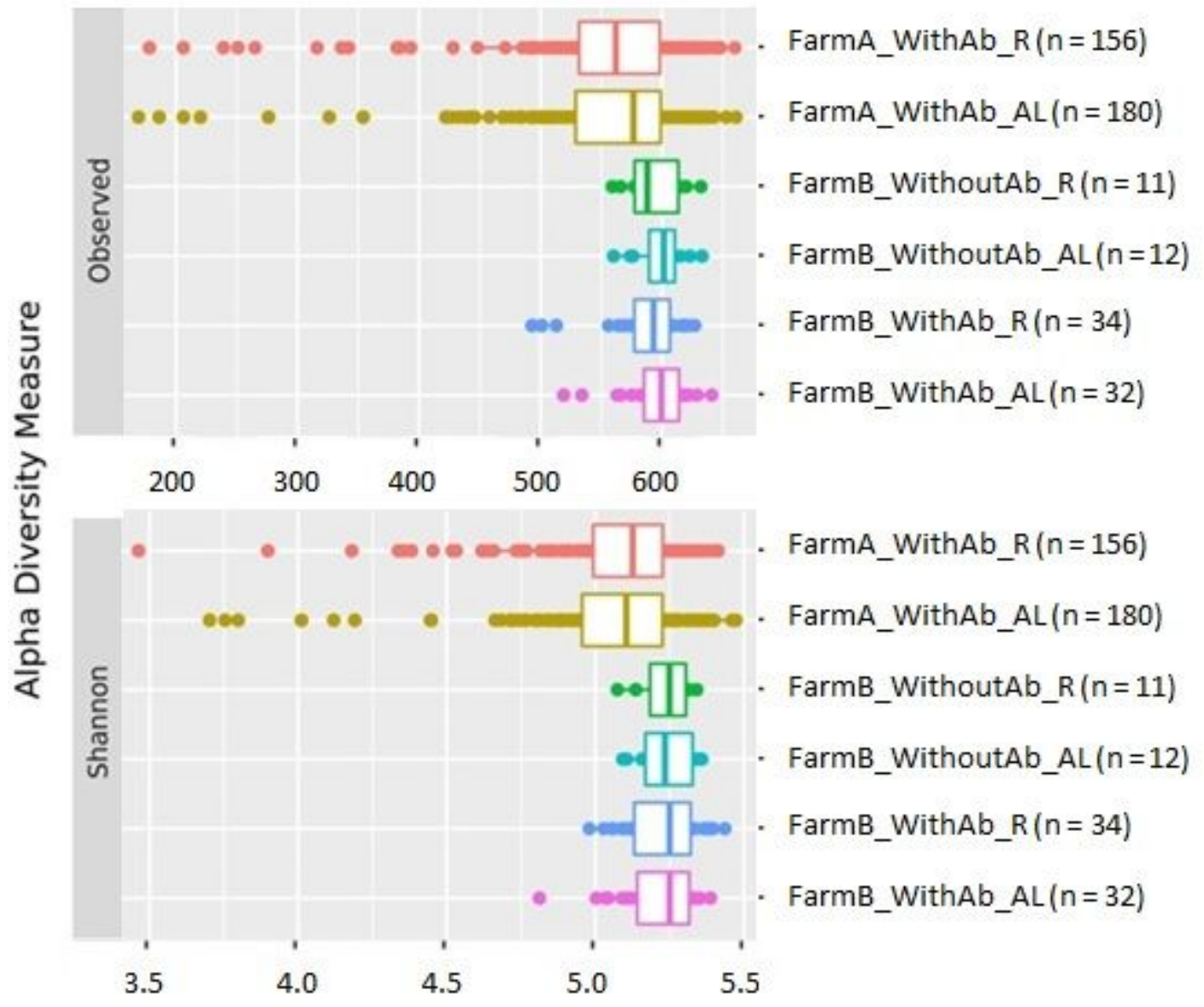

Figure 2

Microbial richness and diversity between samples grouped according to management that animals received. 


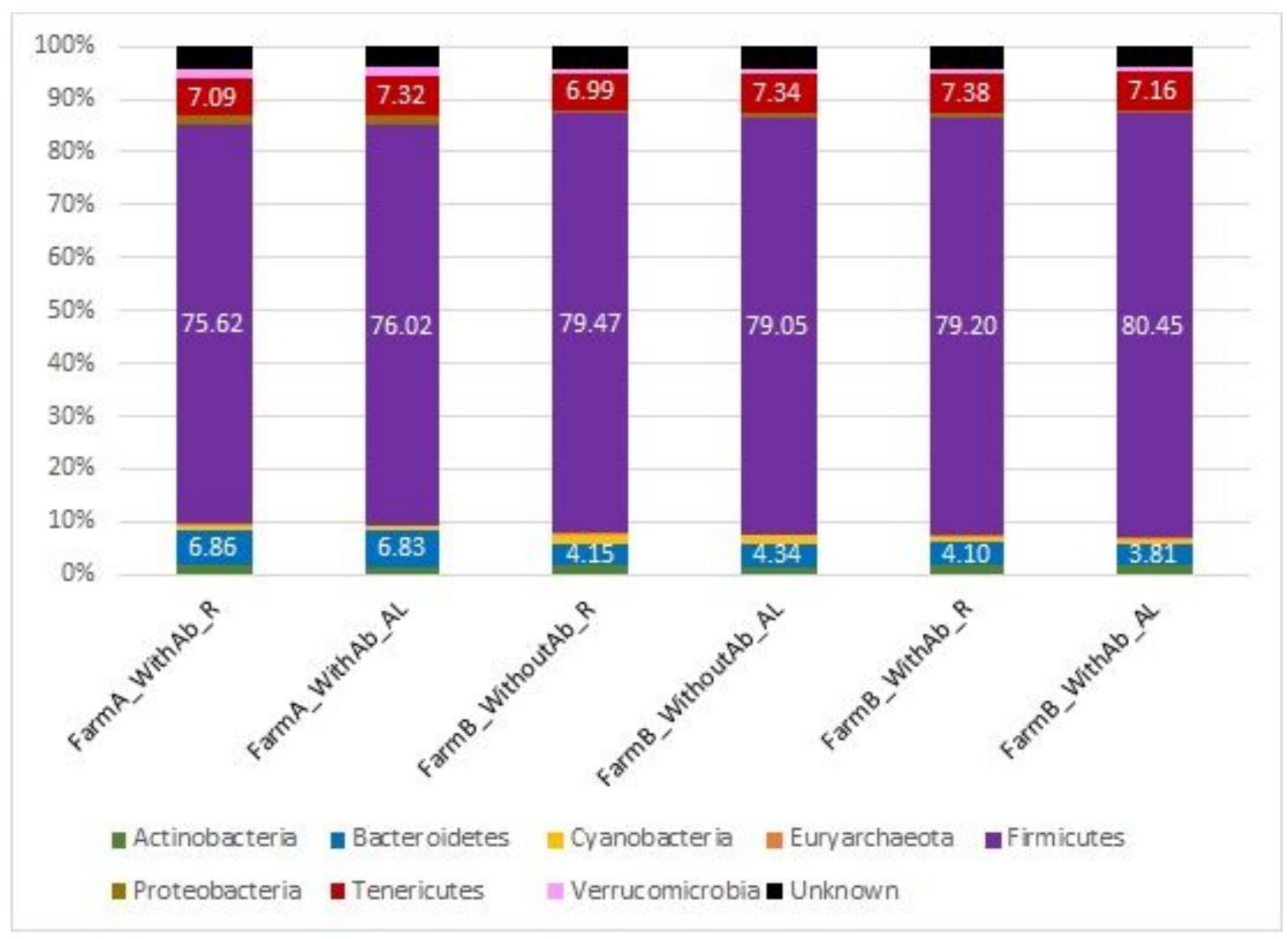

Figure 3

Phyla relative abundances of samples grouped according to farm, level of feeding and presence of antibiotics in the feed. 


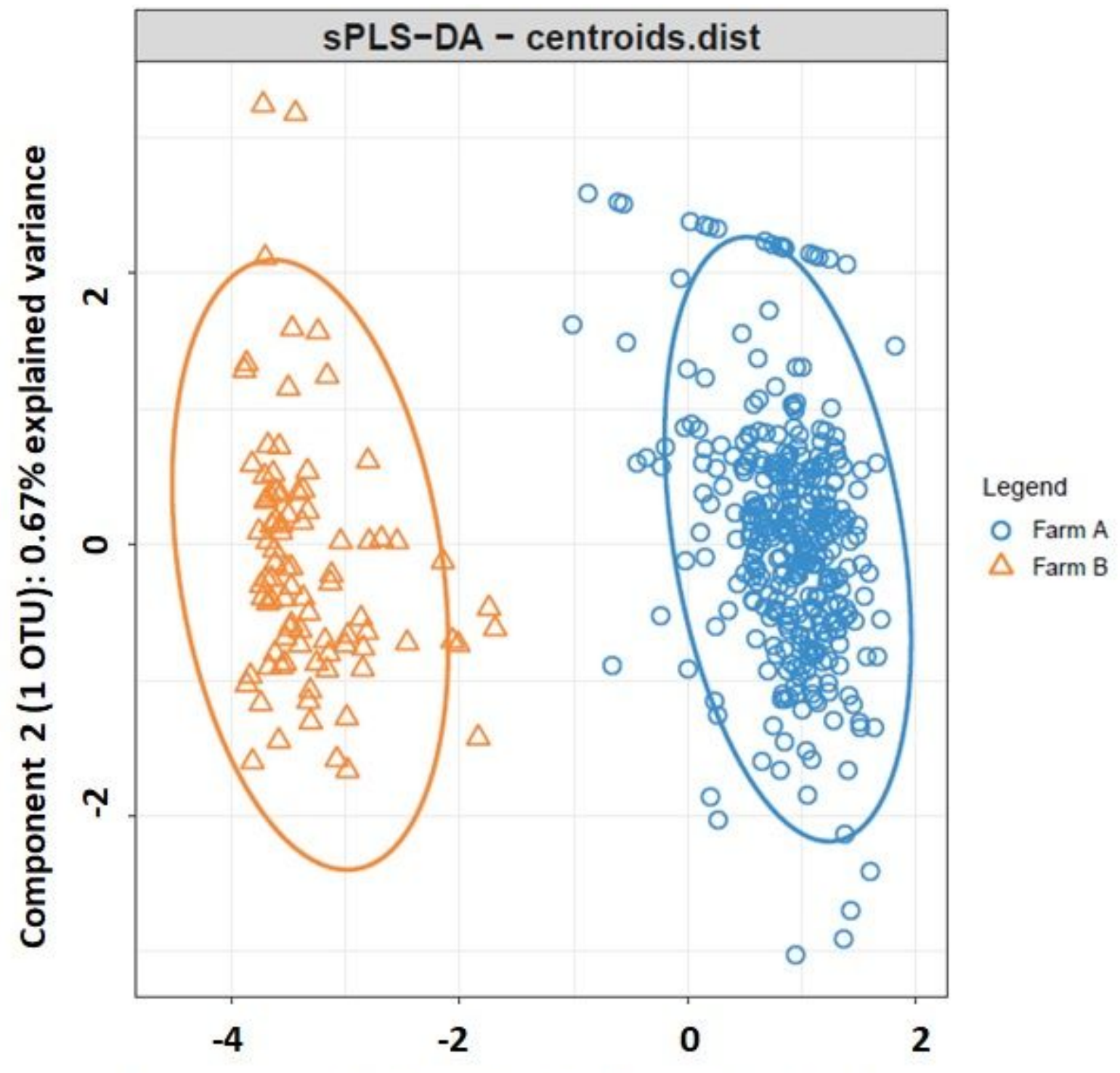

Component 1 (5 OTUs): $7.00 \%$ explained variance

Figure 4

Sparse partial least squares discriminant analysis representing cecal samples of rabbits raised in farm $A$ (blue) and in farm B (orange). 


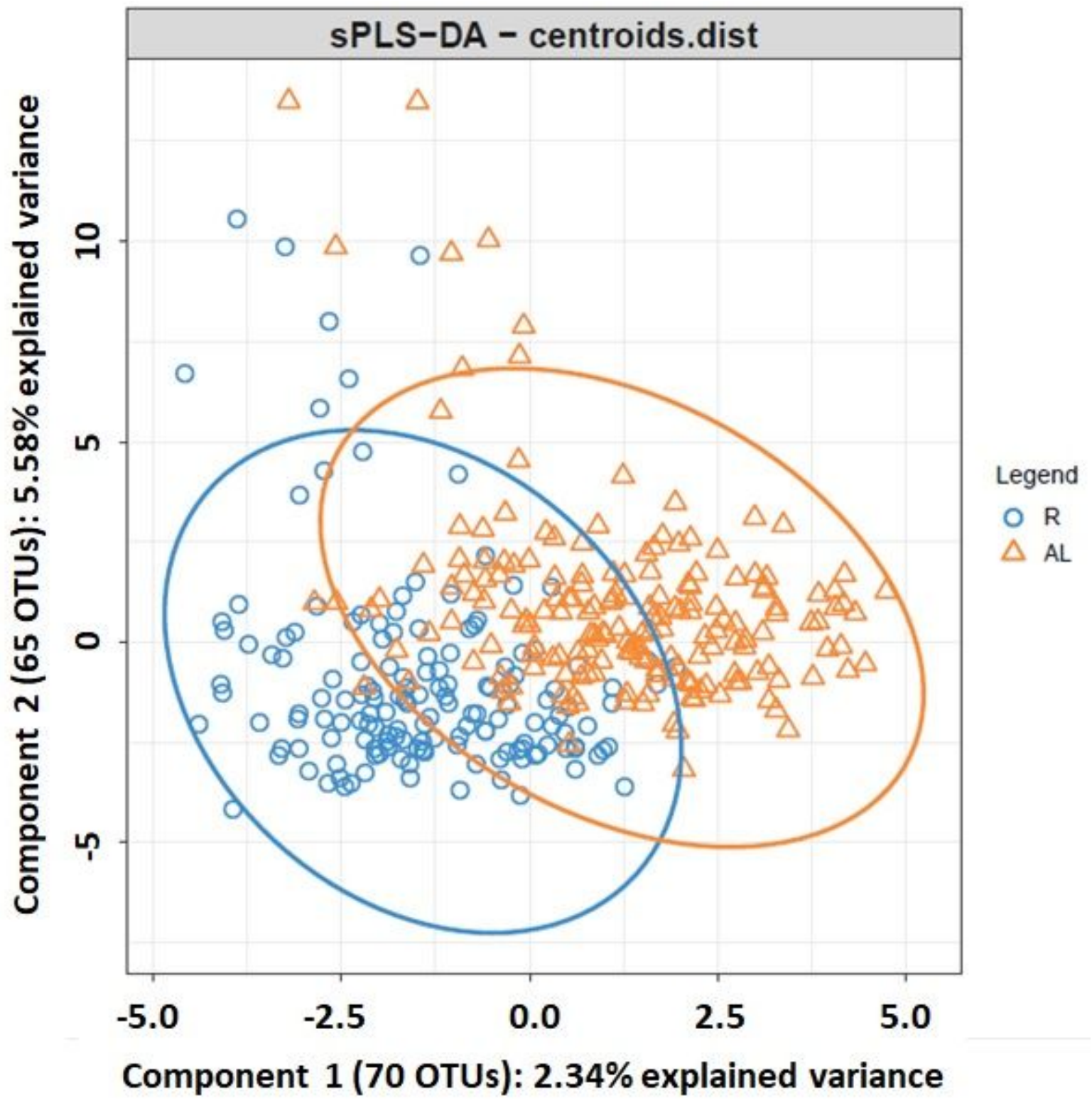

Figure 5

Sparse partial least squares discriminant analysis representing cecal samples of rabbits raised in farm A and fed $\mathrm{R}$ (blue) or $\mathrm{AL}$ (orange). 


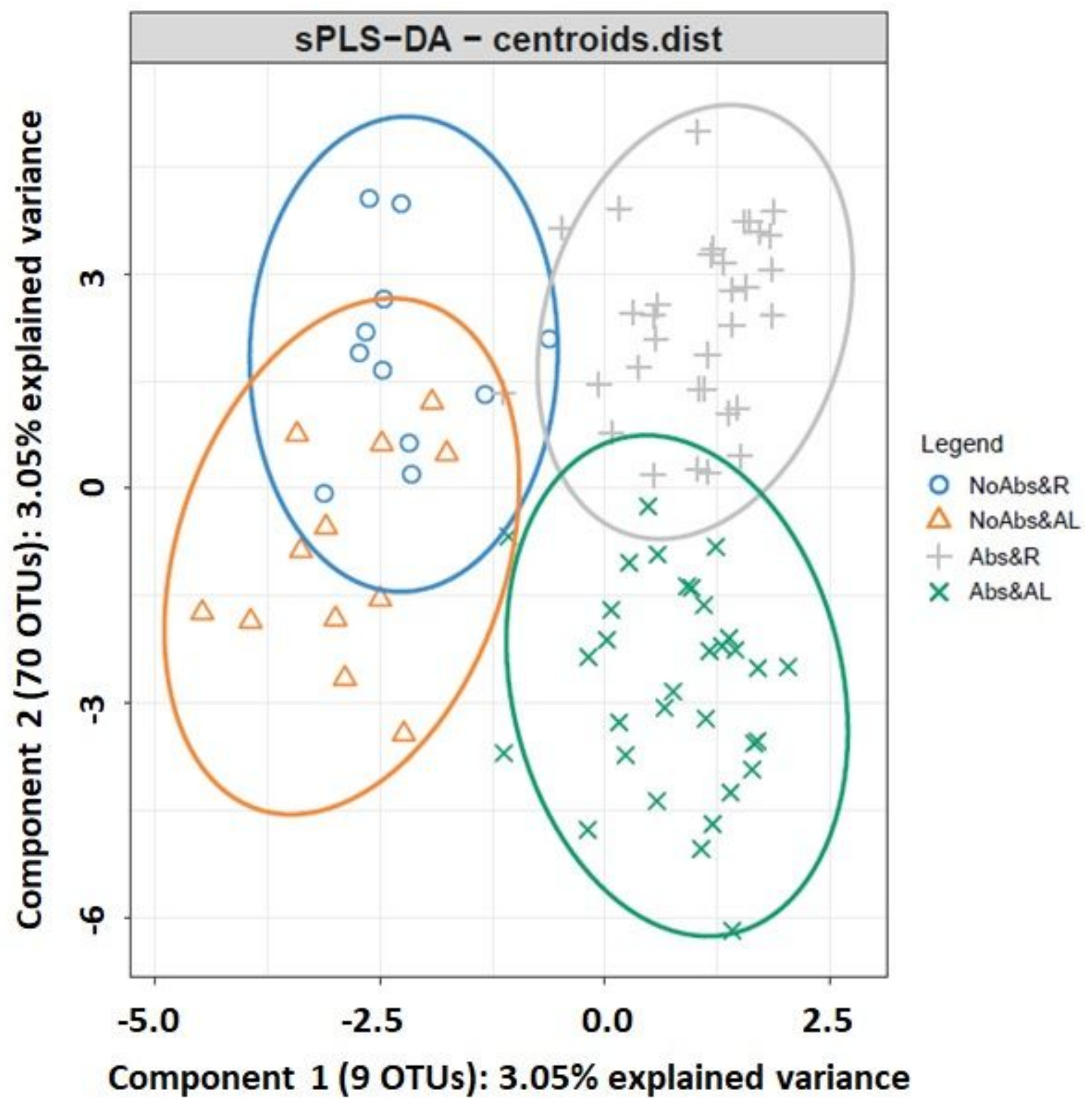

Figure 6

Sparse partial least squares discriminant analysis representing cecal samples of rabbits raised in farm B and fed R withoutAb (blue), fed AL withoutAb (orange), fed R withAb (gray) and fed AL withAb (green).

\section{Supplementary Files}

This is a list of supplementary files associated with this preprint. Click to download.

- repOTUstaxassignments.txt.txt

- phylatable.txt.txt

- otutableprefilteredunnormalized.txt.txt

- otutablefilteredCSSnormalized.txt.txt 
- metadata.txt.txt

- generatable.txt.txt

- OTUsdiffsregime.xIsx

- OTUsdiffsfarm.xIsx

- OTUsdiffantibiotics.xIsx 